\title{
ON HYPERBOLIC COBORDISMS AND HURWITZ CLASSES OF HOLOMORPHIC COVERINGS
}

\begin{abstract}
CARLOS CABRERA, PETER MAKIENKO, AND GUILLERMO SIENRA
ABSTRACT. In this article we show that for every collection $\mathcal{C}$ of an even number of polynomials, all of the same degree $d>2$ and in general position, there exist two hyperbolic 3-orbifolds $M_{1}$ and $M_{2}$ with a Möbius morphism $\alpha: M_{1} \rightarrow M_{2}$ such that the restriction of $\alpha$ to the boundaries $\partial M_{1}$ and $\partial M_{2}$ forms a collection of maps $Q$ in the same conformal Hurwitz class of the initial collection $\mathcal{C}$. Also, we discuss the relationship between conformal Hurwitz classes of rational maps and classes of continuous isomorphisms of sandwich products on the set of rational maps.
\end{abstract}

\section{INTRODUCTION}

Cobordism theory has been studied widely since it was introduced by H. Poincaré in the context of homology theory. Also R. Thom studied cobordism of embeddings. Since then there has been an interest in cobordism of functions, for instance functions with stable singularities. Cobordims can be endowed with geometric structures such as symplectic structures, flat connections, or complex structures.

For example, start with a pair of Kleinian groups $\Gamma_{1}$ and $\Gamma_{2}$ such that $\Gamma_{1}$ is a subgroup of finite index in $\Gamma_{2}$. The inclusion map generates a Möbius morphism $\alpha: M\left(\Gamma_{1}\right) \rightarrow M\left(\Gamma_{2}\right)$ which is a finite degree orbifold covering. Since $\partial M\left(\Gamma_{1}\right)$ may be disconnected, the restriction $f:=\left.\alpha\right|_{\partial M\left(\Gamma_{1}\right)}$ forms a collection of finite degree holomorphic coverings from the components of $\partial M\left(\Gamma_{1}\right)$ to the components of $\partial M\left(\Gamma_{2}\right)$. In this situation, it is natural to say that the collection $f$ forms a hyperbolic cobordism.

With this point of view we avoid the homological language and will be interested in the following inverse problem.

Given a collection $Q$ of holomorphic finitely degree (orbifold) coverings, does there exists a pair of Kleinian groups and a Möbius morphism $\alpha$ which is conformally equivalent to the collection $Q$ ?

Another motivation to this question is the relational dictionary between rational maps and Kleinian groups. For this reason, the collection of maps will be often taken as a collection of rational endomorphisms of the Riemann sphere. The main results of this article are Theorems 1 and 3 below; these are proven in Sections 3 . 4 and 5 .

In the last section, we characterize algebraically when two rational maps define the same conformal Hurwitz class. Also we briefly remark that the Hurwitz class of a rational map $R$ can be presented as a space of quasiconformal deformations of a semigroup of holomorphic correspondences and discuss the related questions.

Received by the editors January 15, 2019, and, in revised form, October 25, 2019.

2010 Mathematics Subject Classification. Primary 30F40, 32Q45, 37F30, 57M12.

This work was partially supported by PAPIIT IN102515 and CONACYT CB15/255633. 
From now on our surfaces are compact surfaces with finitely many punctures which admit a hyperbolic (orbifold) structure of finite-type. However, some of our results can be extended to the case of infinite-type.

We start with the following definition.

Definition. A branched covering of finite degree $d$ is a triplet $\left(R, S, S^{\prime}\right)$ where $S$ and $S^{\prime}$ are finite collections of Riemann surfaces and $R: S \rightarrow S^{\prime}$ is a continuous surjective mapping so that

(1) If $Y \subset S$ is a component, then $Z=R(Y) \subset S^{\prime}$ is also a component and the restriction $R: Y \rightarrow Z$ is a degree $d_{Y} \leq d$ branched covering map.

(2) There is a component $\tilde{Y}$ such that $d_{\tilde{Y}}=d$.

We say that a branched covering $R$ is simple whenever the number of components of $S$ coincides with the number of components of $S^{\prime}$. If $R$ is simple and $S$ is connected, then we say that $R$ is a single branched covering.

Branched coverings between Riemann surfaces have been studied widely in the literature. We are interested in the following basic examples.

(1) Rational maps, these are single branched self-coverings of the sphere.

(2) Let $\Gamma$ be a Kleinian group and let $G<\Gamma$ be a subgroup with $\Omega(G)=\Omega(\Gamma)$; then the natural projection $R: S(G) \rightarrow S(\Gamma)$ is a holomorphic branched covering map. When $\Omega(G) \neq \Omega(\Gamma)$, in general, the inclusion map does not induce a holomorphic covering. Here $\Omega(\Gamma)$ denotes the discontinuity set of $\Gamma$.

It is known that the equality $\Omega(G)=\Omega(\Gamma)$ holds when either $G$ is a subgroup of finite index or is a non-elementary normal subgroup. However, there are examples of non-elementary groups $G$ with $\Omega(G)=\Omega(\Gamma)$ but such that $G$ is not normal and has infinite index in $\Gamma$. If $G$ and $\Gamma$ are finitely generated and the limit set of $\Gamma$ is not a subset of a round circle, then by the Ahlfors finiteness theorem $G$ necessarily has finite index in $\Gamma$.

Even more, as pointed out by the referee, again by the Ahlfors finiteness theorem $G<\Gamma$ has finite index whenever $\Gamma$ has an invariant component in $\Omega(\Gamma)=\Omega(G)$ for finitely generated groups $G$ and $\Gamma$.

Any branched covering can be regarded as a collection of single coverings; we call each of them a single component of the branched covering. We say that a branched covering $R$ is a holomorphic covering whenever every single component is a holomorphic (orbifold) unbranched covering between hyperbolic surfaces (orbifolds).

Given a holomorphic covering $\left(R, S, S^{\prime}\right)$, we can improve $\left(R, S, S^{\prime}\right)$ into a simple covering in the following way: if $X$ and $Y$ are components of $S$ so that $R(X)=$ $R(Y)=Z \subset S^{\prime}$, then consider a conformal copy $Z^{\prime}$ of $Z$. Let $R^{\prime}: X \rightarrow Z^{\prime}$ be the respective holomorphic covering; now the holomorphic covering $X \sqcup Y \rightarrow R^{\prime}(X) \sqcup$ $R(Y)$ is simple. By induction on the number of components we construct a simple holomorphic covering $\left(Q, T, T^{\prime}\right)$ such that for every single component $R: X \rightarrow Z$ of $\left(R, S, S^{\prime}\right)$ there exists a single component $Q: X^{\prime} \rightarrow Z^{\prime}$ of $\left(Q, T, T^{\prime}\right)$ and two conformal homeomorphisms $\phi: Z \rightarrow Z^{\prime}$ and $\psi: X \rightarrow X^{\prime}$ so that $\phi \circ R=Q \circ \psi$. The previous discussion also motivates the following definition of Hurwitz classes for non-connected branched coverings.

Definition. Let $f: S \rightarrow S^{\prime}$ be a branched covering, let the Hurwitz class $H(f)$ of $f$ consist of the triples $\left(g, N, N^{\prime}\right)$ so that $g: N \rightarrow N^{\prime}$ is a branched covering, and 
let there exist orientation preserving homeomorphisms $\varphi: S \rightarrow N$ and $\psi: S^{\prime} \rightarrow N^{\prime}$ such that $\psi \circ f=g \circ \varphi$.

If $f: S \rightarrow S^{\prime}$ is a single branched covering, then $H(f)$ coincides with the classical Hurwitz space of $f$. If $f$ is a simple branched covering, then

$$
H(f)=\bigotimes_{Y} H(f, Y, f(Y))
$$

where the product is taken over the connected components $Y$ of $S$.

Given a holomorphic map $f$, the set

$$
C H(f)=\left\{\left(g, N, N^{\prime}\right) \in H(f), \phi, \psi \text { conformal }\right\}
$$

is called the conformal Hurwitz class of the holomorphic covering $f$.

For example, if $f: \overline{\mathbb{C}} \rightarrow \overline{\mathbb{C}}$ is a finite degree branched covering, then $H(f)$ contains a rational map of the same degree. When $f$ is a rational map the set $H(f) \cap \operatorname{Rat}(\mathbb{C})=S(f)$ is known as the Speisser class of $f$ and was introduced into holomorphic dynamics by A. Erëmenko and M. Lyubich in [4. By Teichmüller's theorem, if $f$ is holomorphic of finite degree and if $g \in H(f)$ is holomorphic, then the associated homeomorphisms $\phi$ and $\psi$ can be taken quasiconformal. For general holomorphic maps, this is not true. It is not even clear whether $\phi$ and $\psi$ can be taken to be local quasiconformal maps for an infinite degree holomorphic map $f$. A plausible counterexample is an entire map $f$ such that the set of singularities of $f^{-1}$ has positive Lebesgue measure.

The hyperbolic cobordism between two holomorphic coverings $\left(R_{1}, S_{1}, S_{1}^{\prime}\right)$ and $\left(R_{2}, S_{2}, S_{2}^{\prime}\right)$ is given by the triple $\left(\Re, M, M^{\prime}\right)$ satisfying the following conditions:

(1) There are geometrically finite Kleinian groups $\Gamma, \Gamma^{\prime}<P S L(2, \mathbb{C})$ such that

$$
M=M(\Gamma)=(B \bigcup \Omega(\Gamma)) / \Gamma
$$

and

$$
M^{\prime}=M\left(\Gamma^{\prime}\right)=\left(B \bigcup \Omega\left(\Gamma^{\prime}\right)\right) / \Gamma^{\prime} .
$$

Hence, $M$ and $M^{\prime}$ are oriented hyperbolic 3-orbifolds with natural projections $\pi: B \bigcup \Omega(\Gamma) \rightarrow M$, and $\pi^{\prime}: B \bigcup \Omega\left(\Gamma^{\prime}\right) \rightarrow M^{\prime}$. The map $\Re: M \rightarrow M^{\prime}$ is a surjective Möbius morphism, that is, there exists an orientation preserving Möbius map $\alpha$ such that the following diagram commutes:

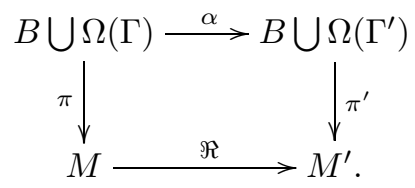

(2) The boundary $\partial M$ is conformally equivalent to $\bigsqcup S_{i}$ and $\partial M^{\prime}$ conformally equivalent to $\bigsqcup S_{i}^{\prime}$, so that

$$
\left(\left.\Re\right|_{\partial M}, \partial M, \partial M^{\prime}\right) \in \bigotimes_{i=1}^{2} C H\left(R_{i}, S_{i}, S_{i}^{\prime}\right) .
$$


Hence $\Re$ is a local isometry between the respective hyperbolic metrics on $M(\Gamma)$ and $M\left(\Gamma^{\prime}\right)$ induced by the Kleinian groups $\Gamma$ and $\Gamma^{\prime}$, respectively.

Given two holomorphic coverings $R_{1}$ and $R_{2}$, if there exists a hyperbolic cobordism between $R_{1}$ and $R_{2}$, we will say that $R_{1} \sqcup R_{2}$ forms a cobordant family of holomorphic coverings, or that $R_{1}$ is hyperbolically cobordant to $R_{2}$.

Given a finite degree holomorphic branched covering $R: M \rightarrow N$, between Riemann surfaces $M$ and $N$, there are many ways to transform $R$ into a holomorphic covering between hyperbolic orbifold structures supported on $M$ and $N$.

We consider the simplest construction depending on the ramification data of $R$ and a finite subset $A \subset N$ as follows: first restrict $R$ to $R:\left\{S=M \backslash R^{-1}(A)\right\} \rightarrow$ $\left\{S^{\prime}=N \backslash A\right\}$. Second, using the ramification data of $R$ produce orbifold structures on $S$ and $S^{\prime}$ so that $R$ is a holomorphic (orbifold) covering between hyperbolic orbifold structures supported on $S$ and $S^{\prime}$, respectively.

In particular, if $A=\emptyset$, then the canonical orbifold structure on $M$ and $N$ defined by the ramification data of $R$ must be hyperbolic. For instance, in the case where $R(z)=z^{n}$, the set $A$ must be non-empty and $\operatorname{card}(A \backslash\{0, \infty\}) \geq 1$ for $n \geq 3$.

If $A=V(R)$ is the set of critical values of $R$, and the surfaces $S$ and $S^{\prime}$ are hyperbolic, then the triple $\left(R, S, S^{\prime}\right)$ is called the canonical holomorphic representative of the holomorphic branched covering $R: M \rightarrow N$.

Examples. (1) The null cobordism where $S$ and $S^{\prime}$ are connected is related to the extension of a single holomorphic covering to the respective 3-hyperbolic spaces. This situation has been studied in [3] with applications to holomorphic dynamical systems. In particular, in [3] the authors gave the construction of a geometric extension for generic rational maps. The present article develops the geometrical part of 3 in the case of a collection of holomorphic coverings.

(2) The trivial cobordisms. Consider the identity maps $\operatorname{Id}_{i}: S_{i} \rightarrow S_{i}$, where $S_{i}$ is a Riemann surface for $i=1,2$. Then the existence of a cobordism between $\operatorname{Id}_{1}$ and $\mathrm{Id}_{2}$ reduces to the existence of a hyperbolic manifold with boundary conformally equivalent to $S_{1} \sqcup S_{2}$. Then we have:

- If $S_{1}$ is quasiconformally equivalent to $S_{2}$, then by the Bers simultaneous uniformization theorem there exists a quasifuchsian group uniformizing the surfaces $S_{1}$ and $S_{2}$, so that $S_{1} \sqcup S_{2}$ is conformally equivalent to the boundary of a hyperbolic 3-manifold. The quasifuchsian group can be chosen a fuchsian group if there is an anticonformal isomorphism between $S_{1}$ and $S_{2}$.

- For any surface $S_{1}$ consisting of a finite number of hyperbolic components, there exists a connected hyperbolic surface $S_{2}$ such that $S_{1} \sqcup S_{2}$ can be uniformized by a geometrically finite function group. This uniformization is given by the Klein-Maskit combination theorems in such a way that $S_{1} \sqcup S_{2}$ bounds an oriented hyperbolic 3-orbifold. This observation will be needed in the proof of Theorem 1 .

- Whenever $S$ is a compact hyperbolic closed connected Riemann surface with an even number of cusps, there exists a Schottky-type group uniformizing $S$ so that $S$ is conformally equivalent to the boundary of a 3-hyperbolic manifold.

Connected transitivity. Given three single holomorphic coverings $R_{1}, R_{2}$, and $R_{3}$ such that the pairs $\left(R_{1}, R_{2}\right)$ and $\left(R_{2}, R_{3}\right)$ are each hyperbolically cobordant by 
manifolds $M_{1}$ and $M_{2}$, and assuming that the canonical homorphisms $\pi_{1}\left(S_{i}\right) \rightarrow$ $\pi_{1}\left(M_{i}\right)$ and $\pi_{1}\left(S_{i}^{\prime}\right) \rightarrow \pi_{1}\left(M_{i}^{\prime}\right)$ are injective, then $R_{1}$ is hyperbolically cobordant to $R_{3}$. In fact, this follows from Thurston's hyperbolization theorem.

In general, without the single and injective assumptions, it is not clear that the manifold, resulting by gluing $M_{1}$ and $M_{2}$ along the boundary components associated to $R_{2}$, is hyperbolic. This is because the result of gluing hyperbolic manifolds along the boundary may not be hyperbolic. For instance, consider a geometrically finite hyperbolic 3-manifold $M$ which has an essentially embedded annulus $A$. Let $S_{1}$ and $S_{2}$ be not necessarily different components of $\partial M$ containing the boundary of $A$. Take a copy of $M$, say $M^{\prime}$, and make $V=M \sqcup_{S_{1} \sqcup S_{2}} M^{\prime}$ by gluing $M$ and $M^{\prime}$ along $S_{1} \sqcup S_{2}$. Then $V$ does not accept a hyperbolic metric since $V$ contains a torus which is not homotopic to the ideal boundary of $V$. This indicates that there might be obstacles to the existence of a hyperbolic cobordism between multiple coverings.

Now we formulate our first main theorem.

Theorem 1. Given a simple holomorphic covering $F_{1}$, there exists another holomorphic covering $F_{2}$ such that $F_{1} \sqcup F_{2}$ forms a family of cobordant holomorphic coverings.

Moreover, if $F_{1}$ has a single component $R_{0}$ with degree $\operatorname{deg}\left(R_{0}\right)>1$, then the covering $F_{2}$ contains only one single component, say $Q_{0}$, with degree larger than 1 and $\operatorname{deg}\left(Q_{0}\right)=\sum_{i=0}^{n} \operatorname{deg}\left(R_{i}\right)-n$ where $R_{i}$ are single components of $F_{1}$ and $n+1$ is the number of these components.

We need the following definition.

Definition. A holomorphic covering $Q: S \rightarrow S^{\prime}$ is called an anticonformal copy of the holomorphic covering map $R: T \rightarrow W$ if there are anticonformal homeomorphisms $\alpha: S \rightarrow T$ and $\beta: S^{\prime} \rightarrow W$ so that $\beta \circ Q=R \circ \alpha$. Given a holomorphic covering $R$, we call the Hurwitz class $H(R)$ symmetric if and only if $H(R)$ contains an anticonformal copy of an element $g \in H(R)$. Finally, we say that a holomorphic covering is symmetric if its Hurwitz class is symmetric.

Let us note that if $f: \overline{\mathbb{C}} \rightarrow \overline{\mathbb{C}}$ is a branched covering, then $H(f)$ is symmetric whenever $H(f)$ contains a real rational map, that is, all coefficients are real. In particular, if $B$ is a Blaschke endomorphism, then $H(B)$ is symmetric. Moreover, if $\bigsqcup_{B} H(B)$ is the union of all Hurwitz classes of Blaschke endomorphisms and $g \in \bigsqcup_{B} H(B)$ then $H(g)$ is symmetric. Also for every natural number $d$, by the Theorem 3.4 in [1], the set $\bigsqcup_{B} H(B) \cap \operatorname{Rat}_{d}(\mathbb{C})$ is connected and contains an open and everywhere dense subset of the space of $\operatorname{Rat}_{d}(\mathbb{C})$. Here $\operatorname{Rat}_{d}(\mathbb{C})$ denotes the set of rational maps of degree $d$. A Blaschke endomorphism is a rational map $B$ with $B^{-1}(\mathbb{D})=\mathbb{D}$ where $\mathbb{D}$ is the open unit disk in $\mathbb{C}$.

In general it is not clear that the Hurwitz class of any finite degree branched covering between closed Riemann surfaces is symmetric. But we believe that is true for Hurwitz classes of rational maps.

Definition. Two cobordant holomorphic coverings $R_{1}$ and $R_{2}$ are called simply cobordant if and only if $M$ and $M^{\prime}$ are homeomorphic to $S_{1} \times[0,1]$ and $S_{1}^{\prime} \times[0,1]$, respectively. 
Theorem 2. Two symmetric single holomorphic coverings $R_{1}$ and $R_{2}$ belong to the same Hurwitz class if and only if $R_{1}$ and $R_{2}$ are simply cobordant.

Theorem 1 shows that any finite family of coverings can be included in a family of cobordant coverings which is non-simple and includes single univalent components on the boundary. Theorem 2 gives a condition for when a pair of holomorphic coverings of the Riemann sphere is cobordant.

With Theorem 2 at hand we improve Theorem 1 into Theorem 3 . First recall that a holomorphic polynomial map $P: \mathbb{C} \rightarrow \mathbb{C}$ of degree $d>1$ is in general position if there are $d-1$ different finite critical values $V(P)$. It is known that two polynomial maps in general position belong to the same Hurwitz class if and only if these polynomials have the same degree. Also, every polynomial in general position is symmetric.

Theorem 3. The canonical holomorphic representatives of every collection of an even number of polynomials in general position of the same degree $d>2$ form a hyperbolic cobordism.

\section{Some BACKGround on KLEINIAN GRoups}

For the convenience of the reader here we collect some facts from Kleinian group theory which will be used in this article. We follow the books of M. Kapovich [5] and A. Marden 6] which give a modern introduction to Kleinian groups.

Denote by $B$ the Poincaré model of the hyperbolic 3 -space, that is, the unit ball in $\mathbb{R}^{3}$ equipped with the Poincaré metric. Given a group $\Gamma$ of automorphisms of the Riemann sphere, we denote by $\Omega(\Gamma)$ the discontinuity set of $\Gamma$ on $\overline{\mathbb{C}}$. The isometry group of $B$ acts on the Riemann sphere $\overline{\mathbb{C}}=\partial B$ as the whole group of Möbius transformations $\operatorname{Mob}(\mathbb{C})$ including anticonformal automorphisms. A discrete subgroup $\Gamma$ of $\operatorname{Isom}(B)$ is a Kleinian group if $\Omega(\Gamma) \neq \emptyset$. Historically, a Kleinian group is defined as a subgroup of orientation preserving isometries of $B$, but we need the extended definition in order to apply Brook's deformation theorem. Also we follow the definition in [5] where it is shown that many classical theorems for orientation preserving Kleinian groups extend to the general case without many difficulties.

Define $S(\Gamma)=\Omega(\Gamma) / \Gamma$ and $M(\Gamma)=(B \sqcup \Omega(\Gamma)) / \Gamma$ and note that $S(\Gamma)=\partial M(\Gamma)$.

Both spaces $S(\Gamma)$ and $M(\Gamma)$ can be endowed with a hyperbolic orbifold structure. For an orbifold $O$, let $|O|$ be the underlying space of $O$. When $\Gamma$ contains orientation reversing elements, one has to be cautious with the fact that $|S(\Gamma)|$ is a proper subset of $\partial|M(\Gamma)|$. The points in $\partial|M(\Gamma)| \backslash|S(\Gamma)|$ are interior points in the orbifold structure contained in the singular locus. In other words, neighborhoods of these points are modeled by the quotient of a ball by the action of a finite group of isometries of $B$. The simplest example to have in mind is the space $X$ which is the quotient of $\mathbb{C}$ by the map $z \mapsto \bar{z}$. Then $X$ admits the structure of a manifold with boundary homeomorphic to the closure of the upper half-space. Alternatively, $X$ also possesses the structure of an orbifold without boundary where the real line is the singular locus of the orbifold.

Definition. A 3-manifold $M$ is called geometrically finite if there exists a compact submanifold with boundary $M_{0}$ such that $M \backslash M_{0}$ is a disjoint union of finitely 
many pieces $V_{i}$ such that either

- $V_{i}$ is homeomorphic to $\mathbb{S}^{1} \times(\mathbb{D} \backslash\{0\})$, where $\mathbb{D}$ is the open unit disk in $\mathbb{C}$ and $\mathbb{S}^{1}$ is the unit circle in $\mathbb{C}$; or

- $V_{i}$ is homeomorphic to $[0,1] \times(\mathbb{D} \backslash\{0\})$ so that the punctured disks $\{0\} \times$ $(\mathbb{D} \backslash\{0\})$ and $\{1\} \times(\mathbb{D} \backslash\{0\})$ belong to $\partial M$.

A Kleinian group $\Gamma$ is called geometrically finite if and only if it contains a finite index subgroup $\Gamma_{0}$ such that $M\left(\Gamma_{0}\right)$ is geometrically finite.

The pieces $V_{i}$ in the definition are usually known as solid cusp torii and solid pairing tubes, respectively. There are many equivalent definitions of geometrically finite Kleinian groups; see for example [5] and [6].

2.1. Pinching. In what follows we describe the pinching procedure for a finite family of disjoint simple closed geodesics on a Riemann surface $S$ contained in $S(\Gamma)$ for a geometrically finite Fuchsian group $\Gamma<P S L(2, \mathbb{C})$, according to theorems of B. Maskit [7] and K. Ohshika [10; see also the pinching theorem of Section 5.15 in [6].

For $r>0$ let $A_{r}=\left\{z: \frac{1}{r}<|z|<r\right\}$ be the round symmetric annulus and consider the homeomorphism of the plane $F(z)=z|z|$; note that $F(z)$ is quasiconformal. Take the sequence $\mu_{n}$ of Beltrami differentials on $A_{r}$ defined by $\mu_{n}=\left.\frac{\bar{\partial} F^{n}}{\partial F^{n}}\right|_{A_{r}}$ where $F^{n}$ is the $n$th iterated of $F$ and the partial derivatives are taken in the sense of distributions. Then $\left\|\mu_{n}\right\| \rightarrow 1$ as $n \rightarrow \infty$.

Let $l_{i}$ be a finite collection of disjoint simple closed geodesics in $S$; then by the collar lemma there exists $r_{0}$ and a family of conformal embeddings $h_{i}: A_{r_{0}} \rightarrow$ $S(\Gamma)$ with $h_{i}\left(\mathbb{S}^{1}\right)=l_{i}$ and the closed sets $\overline{h\left(A_{r_{0}}\right)}$ are mutually disjoint. By taking the simultaneous push-forward of $\mu_{n}$ by the maps $h_{i}$, we obtain a sequence $\tilde{\nu}_{n}$ of Beltrami differentials on $S$ supported on the union of the annular neighborhoods $h_{i}\left(A_{r_{0}}\right)$. Now lift the sequence $\tilde{\nu}_{n}$ over $\Omega(\Gamma)$ by the natural projection $\Omega(\Gamma) \rightarrow S(\Gamma)$ to get a sequence $\nu_{n}$ of Beltrami differentials in $\Omega(\Gamma)$ with $\left\|\nu_{n}\right\| \rightarrow 1$. If $f_{n}$ is a solution of the Beltrami equation with coefficient $\nu_{n}$, then the group $\Gamma_{n}=f_{n} \circ \Gamma \circ f_{n}^{-1}$ in $P S L(2, \mathbb{C})$ is quasifucshian. In case that $\Gamma$ acts on $\mathbb{D}$ and $S=\mathbb{D} / \Gamma$ then all the maps $f_{n}$ are holomorphic outside $\overline{\mathbb{D}}$.

Then the following theorem is true.

Theorem 4. Let $\Gamma_{n}$ be a family of quasifuchsian groups as constructed above. After taking a suitable subsequence there exists a geometrically finite Kleinian group $\Gamma_{\infty}=\lim \Gamma_{n_{k}}$ in the topology of convergence on generators so that

- $\Gamma_{\infty} \simeq \Gamma$.

- The interior of $M\left(\Gamma_{\infty}\right)$ is homeomorphic to the interior of $M(\Gamma)$.

- The surface $S\left(\Gamma_{\infty}\right)$ is homeomorphic to $S(\Gamma) \backslash\left(\bigcup l_{i}\right)$. Even more, the homeomorphism can be chosen to be holomorphic outside $\bigcup h_{i}\left(A_{r_{0}}\right)$ and each $l_{i}$ determines a pair of punctures in $S\left(\Gamma_{\infty}\right)$,

The previous theorem is proved by Maskit for function groups, with the condition that the closed simple curves $l \in S(\Gamma)$ to be pinched must have loxodromic representatives in the group, which represent different conjugacy classes. The last condition always holds for simple closed geodesics which belong to the same connected component of $S(\Gamma)$ for a given quasifuchsian group $\Gamma$. In [10, Ohshika extends the theorem of Maskit to all geometrically finite groups. Our version follows the exposition of Marden in the pinching theorem of Section 5.15 of $[6]$. 
Klein-Maskit combination theorem. We will need the following theorem in the proof of Theorem [1, See [7].

Theorem 5 (Klein-Maskit's combination Theorem I [8].). For $i=1,2$, let $\Gamma_{i}$ be a Kleinian group with region of discontinuity $\Omega_{i}$ and a fundamental region $F_{i}$. Assume that there is a simple closed loop $\gamma$ contained in the interior of $F_{1} \cap F_{2}$, bounding two complemented disks $D_{1}$ and $D_{2}$ with $\bar{D}_{i} \subset F_{i}$. Then $\Gamma=\left\langle\Gamma_{1}, \Gamma_{2}\right\rangle$ is a Kleinian group, such that:

(1) The group $\Gamma$ is isomorphic to the free product $\Gamma_{1} * \Gamma_{2}$. If $\Gamma_{1}$ and $\Gamma_{2}$ are geometrically finite, then $\Gamma$ is so.

(2) Let $S_{i}=K_{i} / \operatorname{Stab}\left(K_{i}\right)$ be surfaces where $K_{i} \subset \Omega\left(\Gamma_{i}\right)$ are the components containing $\gamma$ and $\operatorname{Stab}\left(K_{i}\right)<\Gamma_{i}$ are their respective stabilizers. Then $S(\Gamma)$ is homeomorphic to

$$
\left(S\left(\Gamma_{1}\right) \backslash S_{1}\right) \sqcup\left(S\left(\Gamma_{2}\right) \backslash S_{2}\right) \sqcup\left(S_{1} \# S_{2}\right),
$$

where $S_{1} \# S_{2}$ is the connected sum of the surfaces $S_{1}$ and $S_{2}$ along the respective projections of $D_{1}$ and $D_{2}$.

Even more, this homeomorphism can be chosen holomorphic on $S(\Gamma) \backslash$ $S_{1} \# S_{2}$.

(3) The manifold $M(\Gamma)$ is homeomorphic to the disk sum $M\left(\Gamma_{1}\right)$ with $M\left(\Gamma_{2}\right)$ induced by the disks determining the connected sum $S_{1} \# S_{2}$.

Disk patterns and Brook's deformation theorem. The following construction is needed in the proof of Theorem 3 .

Definition. Let $\Gamma$ be a geometrically finite torsion-free Kleinian group. Then a collection $K$ of closed sets $K_{i} \subset S(\Gamma)$ is called a round disk collection if and only if the set $K$ consists of finitely many elements and every element $K_{i}$ is either a homeomorphic projection of a compact round disk $D \subset \Omega(\Gamma)$ to $S(\Gamma)$ or is a closed punctured disk in $S(\Gamma)$ where the puncture corresponds to a cusp of $S(\Gamma)$ and $K_{i}$ is covered by a round disk $D \backslash\{p\} \subset \Omega(\Gamma)$, where $D$ is precisely invariant under its parabolic stabilizer $\gamma \in \Gamma$ and $p$ is the fixed point of $\gamma$ with $p \in \partial D$.

Definition. A finite round disk collection $K \subset S(\Gamma)$ is called a pattern of round disks if and only if the following holds:

(1) No point in $S(\Gamma)$ is covered by the interior of more than two disks in $K$.

(2) Given two different disks $K_{i}$ and $K_{j}$, then either the interiors of $K_{i}$ and $K_{j}$ are disjoint or their boundaries are orthogonal.

Each disk $K_{i} \subset K$ is covered by a round disk $D_{i} \in \overline{\mathbb{C}}$. If $C\left(D_{i}\right)$ is the convex hull of $\partial D_{i}$ in $B$ with respect to the Poincaré metric, then $C\left(D_{i}\right)$ is invariant under the stabilizer of $D_{i}$ in $\Gamma$. Let $V\left(D_{i}\right)$ be the component of $\bar{B} \backslash C\left(D_{i}\right)$ containing $D_{i}$. Let $Y\left(K_{i}\right) \subset M(\Gamma)$ be the projection of $V\left(D_{i}\right)$ in $M(\Gamma)$ and

$$
M_{K}=M(\Gamma) \backslash \bigcup_{K_{i} \in K} Y\left(K_{i}\right)
$$

then on the manifold $M_{K}$ there exists a natural polyhedral geometric structure $\mathcal{G}$ which on the interior of $M_{K}$ coincides with the hyperbolic structure of $\operatorname{int}(M(\Gamma))$, on $M(\Gamma) \cap\left(\bigcup \partial Y\left(K_{i}\right)\right)$ the structure $\mathcal{G}$ is a polyhedral piecewise geodesic structure, and on the remaining boundary components of $M_{K}$ the structure coincides with the Möbius structure inherited from $M(\Gamma)$. 
Let $\tilde{K}$ be the collection of all round disks in $\Omega(\Gamma)$ which cover all $K_{i} \subset K$. Let $\Gamma_{K}<\operatorname{Isom}(B)$ be the group generated by $\Gamma$ and the reflections with respect to the circles $\partial D$ for $D \in \tilde{K}$. Then Theorem 13.1 in [5] states as follows.

Theorem 6. The group $\Gamma_{K}$ is geometrically finite and $M\left(\Gamma_{K}\right)$ is an orbifold diffeomorphic to $M_{K}$ equipped with the structure $\mathcal{G}$.

Remarks. (1) In particular, if a component $\Omega_{0} \subset \Omega(\Gamma)$ does not intersect $\tilde{K}$, then the stabilizer of $\Omega_{0}$ in $\Gamma_{K}$ coincides with the stabilizer of $\Omega_{0}$ in $\Gamma$. Therefore, if a disk pattern $K$ completely covers exactly one component $S \subset$ $\partial M(\Gamma)$, then $\partial\left(M\left(\Gamma_{K}\right)\right)$ is a 2-dimensional orbifold which is conformally equivalent to $\partial M(\Gamma) \backslash S$.

(2) The charts around points on $\partial\left(Y\left(K_{i}\right)\right)$ are modeled with the quotient of the unit 3-dimensional ball by a finite group, this group is generated by reflections on planes passing through the origin. In particular, in this structure the points in $\partial Y\left(K_{i}\right) \backslash K_{i}$ are interior points of $M_{K}$ equipped with the structure $\mathcal{G}$.

The following simple example shows how this procedure works. Let $S$ be any Riemann surface and let $\Gamma$ be a Fuchsian group uniformizing $S$. Then $M(\Gamma)$ is a hyperbolic manifold homeomorphic to $S \times[0,1]$, the boundary of $M(\Gamma)$ consists of $S$ and an anticonformal copy of $S$. Let $\tau$ be the reflection with respect to the unit circle, then $\tau$ commutes with $\Gamma$. Let $G=\langle\Gamma, \tau\rangle$. Thus $G$ is a Kleinian group and $M(G)$ is a non-orientable hyperbolic orbifold, so that $\partial M(G)$ is conformally equivalent to $S$. The underlying space of the orbifold $M(G)$ is a manifold which still is homeomorphic to $S \times[0,1]$ but now, one of the components consists of interior points of the orbifold structure on $M(G)$.

The following theorem justifies the existence of a pattern of disks for a quasiconformal deformation of a given geometrically finite group. This theorem is part of the proof of the Brooks orbifold deformation theorem. More precisely, see the steps 1 to 4 in the proof presented in Section 13.5 of [5]. The statement is as follows.

Theorem 7. For any torsion-free geometrically finite Kleinian group $\Gamma$ there exists a quasiconformal homeomorphism $h$ such that the group $\Gamma_{h}=h \circ \Gamma \circ h^{-1}$ is so that $S\left(\Gamma_{h}\right)$ admits a pattern $K$ which completely covers $S\left(\Gamma_{h}\right)$.

With this background we can proceed to prove our theorems.

\section{Proof of Theorem 1}

3.1. Connected sums of single coverings. Now we reproduce a topological operation between branched coverings which is a sort of "connected sum" of coverings. This operation consists of taking the connected sum of the target surfaces and a pull-back with respect to the branched coverings. Note that there are different ways to make a pull-back. We choose the simplest as follows.

Start with two single finite degree branched coverings $R_{1}: S \rightarrow S^{\prime}$ and $R_{2}$ : $T \rightarrow T^{\prime}$. We construct $R_{0}=R_{1} \# R_{2}$, the connected sum of branched covering maps $R_{1}$ and $R_{2}$, as a branched covering between two surfaces $U$ and $W$ such that $\operatorname{deg}\left(R_{0}\right)=\operatorname{deg}\left(R_{1}\right)+\operatorname{deg}\left(R_{2}\right)-1$ and $W=T^{\prime} \# S^{\prime}$ is the connected sum with respect to topological disks $D_{S} \subset S^{\prime}$ and $D_{T} \subset T^{\prime}$ not containing the branched points (critical values) of $R_{1}$ and $R_{2}$, respectively. 
Topological construction of $U$. Set $\operatorname{deg}\left(R_{1}\right)=n$ and $\operatorname{deg}\left(R_{2}\right)=m$. Let $S^{\prime \prime}=$ $S^{\prime} \backslash D_{S}$ and $T^{\prime \prime}=T^{\prime} \backslash D_{T}$ and $h: \partial D_{S} \rightarrow \partial D_{T}$ be a gluing homeomorphism. Let $S_{0}=R_{1}^{-1}\left(S^{\prime \prime}\right) \subset S$ and $T_{0}=R_{2}^{-1}\left(T^{\prime \prime}\right) \subset T$. Let us fix the following system of curves and homeomorphisms.

(1) Take two components of the boundaries, $\alpha_{0}$ a component of $\partial S_{0}$ and $\beta_{0}$ a component of $\partial T_{0}$. Let $\phi_{0}: \alpha_{0} \rightarrow \beta_{0}$ be a homeomorphism such that $R_{2} \circ \phi_{0}=h \circ R_{1}$.

(2) If $\gamma$ is another component of either $\partial S_{0}$ or $\partial T_{0}$, and different from $\alpha_{0}$ and $\beta_{0}$, then fix a homeomorphism $\phi_{\gamma}$ which is either $\left.h \circ R_{1}\right|_{\gamma}$ or $\left.h^{-1} \circ R_{2}\right|_{\gamma}$, depending on the case. For $i=1, \ldots, m-1$, let $\left\{S_{i}\right\}$ be $m-1$ copies of $S^{\prime \prime}$ and for $j=1, \ldots, n-1$ let $\left\{T_{i}\right\}$ be a family of $n-1$ copies of $T^{\prime \prime}$. Then

$$
U=S_{0} \sqcup T_{0} \sqcup\left\{\sqcup S_{i}\right\} \sqcup\left\{\sqcup T_{i}\right\} / \sim,
$$

where the quotient is taken according to the system of homeomorphisms. More precisely, the homeomorphism $\phi_{0}$ identifies $\alpha_{0}$ with $\beta_{0}$, and the map $\phi_{\gamma}$ identifies the component $\gamma$, which is either in $\partial S_{0}$ or in $\partial T_{0}$, with the respective copies of $T^{\prime \prime}$ or $S^{\prime \prime}$. The identification is taken in such a way that $U$ is a connected surface and there exists a branched covering $R_{1} \# R_{2}: U \rightarrow$ $W$ so that $\left.R_{1} \# R_{2}\right|_{S_{0}}=R_{1}: S_{0} \rightarrow S^{\prime \prime}$ and $\left.R_{1} \# R_{2}\right|_{T_{0}}=R_{2}: T_{0} \rightarrow T^{\prime \prime}$. The restriction of $R_{1} \# R_{2}$ on each one of the remaining copies, of either $T^{\prime \prime}$ or $S^{\prime \prime}$, is univalent.

Then, we have that

$$
\operatorname{genus}(U)=\operatorname{genus}(S)+(m-1) \operatorname{genus}\left(S^{\prime}\right)+\operatorname{genus}(T)+(n-1) \operatorname{genus}\left(T^{\prime}\right) .
$$

Moreover, punctures and holes satisfy the same equation as the genus.

If $R_{1}$ and $R_{2}$ are holomorphic branched coverings between Riemann surfaces, then by taking a conformal gluing in the construction of $U$ we can assume that $R_{1} \# R_{2}: U \rightarrow W$ is a holomorphic branched covering. In other words, the Hurwitz class of a topological connected sum between surfaces contains a holomorphic branched covering.

Hence any branched covering of the Riemann sphere, in general position and degree $d$, can be presented as the connected sum of $d-1$ copies of $z^{2}$.

If $R_{1}(z)=z^{2}$ and $R_{2}(z)=z^{3}$, then $R_{1} \# R_{2}$ is a degree 4 branched self-covering of the topological sphere.

Figure 1 illustrates yet another example; in this case, it shows the connected sum of rational maps in general position of degree 3 and 4 .

3.2. Pinching. We can recover the factors of the connected sum $R_{1} \# R_{2}$ by a pinching procedure as follows.

Let us note that if $R_{1} \# R_{2}: S_{1} \rightarrow S_{2}$ is a connected sum of coverings with $\operatorname{deg}\left(R_{1}\right), \operatorname{deg}\left(R_{2}\right) \geq 2$, then $R_{1} \# R_{2}: U \rightarrow W$ is a finite degree covering between hyperbolic surfaces where

$$
W=S_{2} \backslash \operatorname{CritVal}\left(R_{1} \# R_{2}\right) \text { and } U=S_{1} \backslash\left(R_{1} \# R_{2}\right)^{-1}\left(\operatorname{CritVal}\left(R_{1} \# R_{2}\right)\right) .
$$

In other words, $R_{1} \# R_{2}$ always has a canonical representative whenever

$$
\operatorname{deg}\left(R_{1}\right), \operatorname{deg}\left(R_{2}\right) \geq 2 .
$$




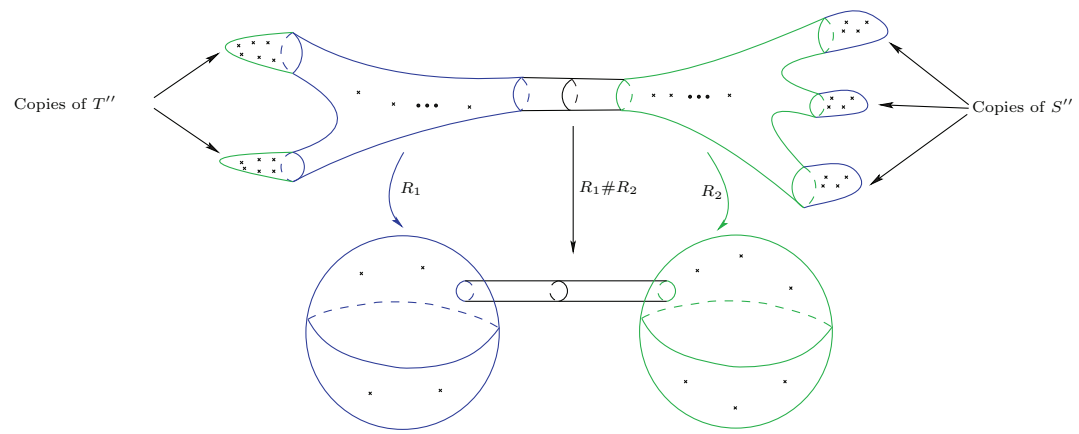

FiguRE 1. The connected sum of rational maps $R_{1}$ and $R_{2}$ of degree 3 and 4 , respectively. The dots depict the respective critical values below and their preimages above.

Let $G, \Gamma_{2}$ be the Fuchsian groups uniformizing the surfaces $U$ and $W$ in the unit disk, respectively. Let $\pi_{U}, \pi_{W}: \mathbb{D} \rightarrow U, W$ be the respective uniformizing projections; then there exists $\alpha$ a Möbius automorphism of $\mathbb{D}$ satisfying $R_{1} \# R_{2} \circ \pi_{U}=$ $\pi_{W} \circ \alpha$ and such that the subgroup $\Gamma_{1}=\alpha G \alpha^{-1}<\Gamma_{2}$ has finite index.

We will say that the fixed pair of groups $\Gamma_{1}<\Gamma_{2}$ and the inclusion map uniformizes $R_{1} \# R_{2}$.

On the other hand, the pair $\Gamma_{1}<\Gamma_{2}$ acts on $\mathbb{D}^{*}$ where $\mathbb{D}^{*}=\overline{\mathbb{C}} \backslash \overline{\mathbb{D}}$ and defines an orbifold covering map $Q: U^{*} \rightarrow W^{*}$, where $U^{*}=\mathbb{D}^{*} / \Gamma_{1}$ and $W^{*}=\mathbb{D}^{*} / \Gamma_{2}$ are the anticonformal copies of $U$ and $W$, respectively, and such that $Q$ is an anticonformal copy of $R_{1} \# R_{2}: U \rightarrow W$.

Let $C$ be the unique simple closed geodesic in the isotopy class of a simple loop on $W$ providing the connected sum. According to Theorem 4

- There exists a sequence $f_{k}$ of quasiconformal automorphisms of the Riemann sphere holomorphic outside the unit disk such that the groups $\Gamma_{2, k}=$ $f_{k} \circ \Gamma_{2} \circ f_{k}^{-1}$ are quasifuchsian groups converging to a Kleinian group $\Gamma_{2, \infty}$ with an invariant component of $\Omega\left(\Gamma_{2, \infty}\right)$.

- The surface $S\left(\Gamma_{2, \infty}\right)$ is homeomorphic to $(W \backslash C) \sqcup W^{*}$ and $C$ determines a pair of punctures on $S\left(\Gamma_{2}, \infty\right)$. Moreover, the homeomorphism can be chosen conformal outside a tubular neighborhood of $C$.

In other words $S\left(\Gamma_{2, \infty}\right)$ is conformally equivalent to

$$
\left(S^{\prime} \backslash\{x\}\right) \sqcup\left(T^{\prime} \backslash\{y\}\right) \sqcup W^{*},
$$

where $x$ and $y$ are the additional cusps determined by $C$. Indeed the perforations $x$ and $y$ are known as accidental cusps (accidental parabolics) which appear in pinching processes. Let $\Gamma_{1, \infty}$ be the respective limit of the groups $f_{k} \circ \Gamma_{1} \circ f_{k}^{-1}$; then $\Gamma_{1, \infty} \cong \Gamma_{1}$. Since $\left(R_{1} \# R_{2}\right)^{-1}(C)$ consists of finitely many simple closed geodesics on $U$, then according to Theorem 4 the surface $S\left(\Gamma_{1, \infty}\right)$ is topologically equivalent to $\left(U \backslash\left(R_{1} \# R_{2}\right)^{-1}(C)\right) \sqcup U^{*}$. These equivalences can be chosen conformal outside tubular neigborhoods of the curves in $\left(R_{1} \# R_{2}\right)^{-1}(C)$. In conclusion, $\Gamma_{1, \infty}<\Gamma_{2, \infty}$ induces a covering map $H: S\left(\Gamma_{1, \infty}\right) \rightarrow S\left(\Gamma_{2, \infty}\right)$ so that the restriction of $H$ to the component associated to $U^{*}$ is in the conformal Hurwitz class of $Q$. Among the restrictions to the other components of $S\left(\Gamma_{1, \infty}\right)$ which are coverings there are only two which have degree larger than one, the other restrictions are univalent. 
The pair of non-univalent coverings belongs to the conformal Hurwitz class of the coverings $R_{1}$ and $R_{2}$.

The result of pinching the manifolds of the example depicted in Figure 1 is shown in Figure 2.

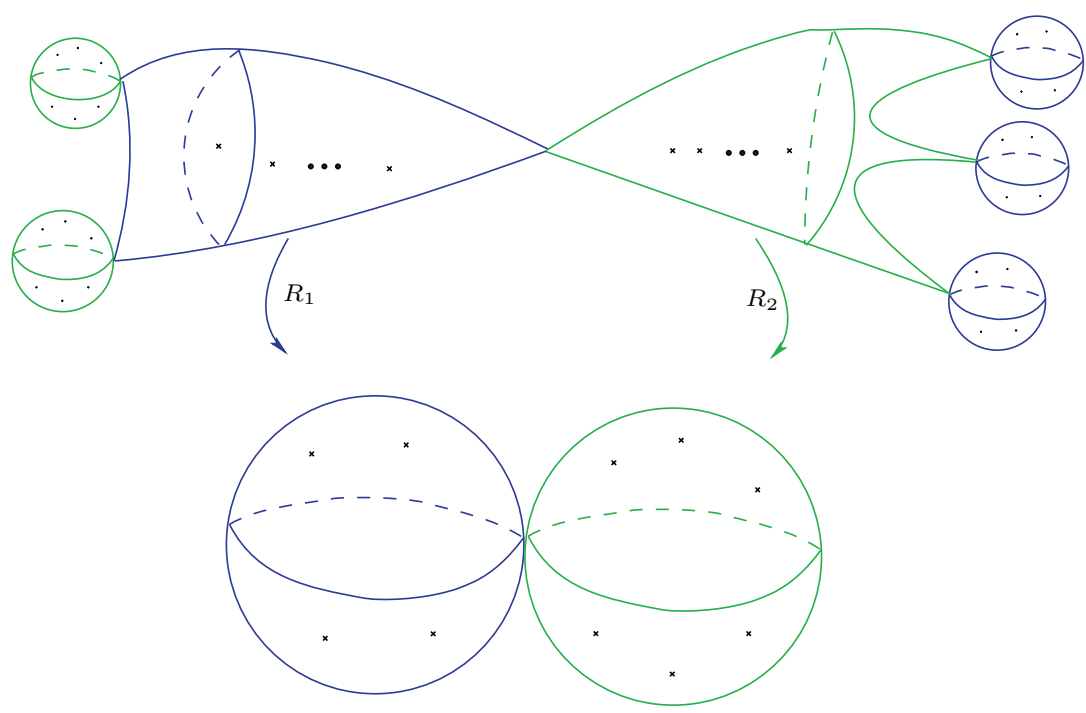

FIGURE 2. In this picture we applied pinching to the example in Figure 1

\subsection{Klein-Maskit combinations and the connected sums of coverings.}

Now we are ready to prove Theorem 1 .

Proof of Theorem 1. First, begin with two single holomorphic covering maps $R_{1}$ : $S \rightarrow S^{\prime}$ and $R_{2}: T \rightarrow T^{\prime}$ of degree $n$ and $m$, respectively, denote by $\Gamma$ and $\Gamma^{\prime}$ the respective Fuchsian uniformizing groups of $S$ and $S^{\prime}$, and groups $G$ and $G^{\prime}$ for the surfaces $T$ and $T^{\prime}$ acting on the unit disk $\mathbb{D}$ so that $\Gamma<\Gamma^{\prime}$ and $G<G^{\prime}$ and the inclusion maps induce the covering $R_{1}$ and $R_{2}$, respectively. If $\mathbb{D}^{*}=\overline{\mathbb{C}} \backslash \overline{\mathbb{D}}$, then inclusion maps $\left(\Gamma, \mathbb{D}^{*}\right) \rightarrow\left(\Gamma^{\prime}, \mathbb{D}^{*}\right)$ and $\left(G, \mathbb{D}^{*}\right) \rightarrow\left(G^{\prime}, \mathbb{D}^{*}\right)$ define anticonformal copies of $R_{1}$ and $R_{2}$, respectively. Denote by $Q_{1}: S^{*} \rightarrow\left(S^{\prime}\right)^{*}$ and $Q_{2}: T^{*} \rightarrow\left(T^{\prime}\right)^{*}$ these anticonformal copies. We have that $\left[\Gamma^{\prime}: \Gamma\right]=n$ and $\left[G^{\prime}: G\right]=m$; following the construction of the previous section we construct a covering map of degree $n+m-1$. Define $R_{0}:=Q_{1} \# Q_{2}$; thus $R_{0}$ maps $U$ to $W=\left(S^{\prime}\right)^{*} \#\left(T^{\prime}\right)^{*}$.

Group construction of $U$. We follow the topological construction above with the Klein-Maskit combination theorem. Let $\tau(z)=1 / \bar{z}$ be the reflection and fix suitable round closed disks $D \subset \tau(F(\Gamma))$ and $\widetilde{D} \subset \tau(F(G))$, where $F(\Gamma)$ and $F(G)$ are fundamental regions for the actions of $\Gamma$ and $G$ in $\mathbb{D}$, respectively. Now let $h \in P S L(2, \mathbb{C})$ be an element so that $h(\partial D)=\partial \widetilde{D}$ and $h$ maps the interior of $D$ onto the exterior of $\widetilde{D}$. Then the pair of groups $\Gamma^{\prime}$ and $h^{-1} \circ G^{\prime} \circ h$ and the disks $D_{1}=D$ and $D_{2}=\overline{\mathbb{C}} \backslash D_{1}$ satisfy the conditions of the Klein-Maskit combination theorem. Since $h^{-1} \circ G^{\prime} \circ h$ is a Möbius copy of $G^{\prime}$ we can assume, by taking suitable Möbius copies, that the groups $\Gamma^{\prime}$ and $G^{\prime}$ already satisfy the conditions of 
Theorem [5. By Theorem 5] the orbit space $S\left(\left\langle\Gamma^{\prime}, G^{\prime}\right\rangle\right)$ is conformally equivalent to $S^{\prime} \sqcup T^{\prime} \sqcup\left(\left(S^{\prime}\right)^{*} \#\left(T^{\prime}\right)^{*}\right)$.

Consider elements $\left\{e, \sigma_{2}, \ldots, \sigma_{n}\right\} \subset \Gamma^{\prime}$ and $\left\{e, g_{2}, \ldots, g_{m}\right\} \subset G^{\prime}$ such that $\Gamma^{\prime}=$ $\Gamma \cup \cdots \cup \sigma_{n} \Gamma$ and $G^{\prime}=G \cup \cdots \cup g_{m} G$. Let $\Gamma_{i}=g_{i} \circ \Gamma^{\prime} \circ g_{i}^{-1}$ be $m-1$ Möbius copies of $\Gamma^{\prime}$ and let $G_{j}=\sigma_{j} \circ G^{\prime} \circ \sigma_{j}^{-1}$ be $n-1$ Möbius copies of $G^{\prime}$. Then by an inductive application of the Klein-Maskit combination theorem the group $H=\left\langle\Gamma, G, \Gamma_{2}, \ldots, \Gamma_{m}, G_{2}, \ldots, G_{n}\right\rangle$ is isomorphic to

$$
\Gamma * G * \prod_{i=2}^{m} \Gamma_{i} * \prod_{j=2}^{n} G_{j} .
$$

Hence, the manifold $M(H)$ is a disk sum of the manifolds $M(\Gamma), M(G), M\left(\Gamma_{i}\right)$, and $M\left(G_{j}\right)$, where the latter are $n-1$ Möbius copies of $M\left(\Gamma^{\prime}\right)$ and $m-1$ Möbius copies of $M\left(G^{\prime}\right)$, respectively.

The inclusion of $H$ in $\Gamma^{\prime} * G^{\prime}$ induces a holomorphic (orbifold) covering

$$
\hat{\iota}: M(H) \rightarrow M\left(\left\langle\Gamma^{\prime}, G^{\prime}\right\rangle\right)
$$

which has finite degree, thus $H$ has finite index in $\left\langle\Gamma^{\prime}, G^{\prime}\right\rangle$. Then the restriction $\hat{\iota}: S(H) \rightarrow S\left(\left\langle\Gamma^{\prime}, G^{\prime}\right\rangle\right)$ is so that there exist three surfaces $S_{1}, S_{2}$, and $S_{3}$ in $S(H)$ where $\operatorname{deg}\left(\left.\hat{\imath}\right|_{S_{j}}\right)>1$, the space $S_{1} \sqcup S_{2} \sqcup S_{3}$ is conformally equivalent to $S \sqcup T \sqcup U$, and $\hat{i}\left(S_{1} \sqcup S_{2} \sqcup S_{3}\right)$ is conformally equivalent to $S^{\prime} \sqcup T^{\prime} \sqcup W$. Moreover, the map $\hat{\iota}$ belongs to $C H\left(R_{1}, R_{2}, Q_{1} \# Q_{2}\right)=C H\left(R_{1}\right) \sqcup C H\left(R_{2}\right) \sqcup C H\left(Q_{1} \# Q_{2}\right)$. If $O \subset S(H) \backslash\left\{S_{1} \sqcup S_{2} \sqcup S_{3}\right\}$, then $\hat{\imath}:=O \rightarrow \hat{\imath}(O)$ is an univalent holomorphic surjective map, even more $\hat{\imath}(O)$ is either $S^{\prime}$ or $T^{\prime}$. So $\hat{\iota}$ is a non-simple holomorphic covering containing single univalent components.

In conclusion, $\hat{\iota}$ is a Möbius morphism which defines a hyperbolic cobordism between the collections $R_{1}: S \rightarrow S^{\prime}, R_{2}: T \rightarrow T^{\prime}, Q_{1}^{\prime} \# Q_{2}: U \rightarrow W$ and single univalent components.

For the general case with three or more coverings $R_{i}: S_{i} \rightarrow S_{i}^{\prime}, i=1, \ldots, k$, we proceed inductively. This finishes the proof.

Now what can we say about non-simple holomorphic coverings? We start with the following examples of uniformizable non-simple holomorphic coverings.

(1) Let $\Gamma$ be a geometrically finite Fuchsian group such that $\gamma \circ \Gamma \circ \gamma^{-1}=\Gamma$ for $\gamma(z)=\frac{1}{z}$. Then $G=\langle\Gamma, \gamma\rangle$ is isomorphic to an $H N N$-extension of $\Gamma$ and is a geometrically finite Kleinian group, so that $M(G)=(\Omega(G) \cup B) / G$ is a geometrically finite hyperbolic orbifold with connected boundary which is a hyperbolic orbifold conformally equivalent to $\mathbb{D} / \Gamma$. More, the inclusion $\Gamma<G$ induces a degree 2 branched covering $\pi: M(\Gamma) \rightarrow M(G)$ so that $\pi(\partial M(\Gamma))=\partial M(G)$ and for any component $S \in \partial M(\Gamma)$ the restriction $\left.\pi\right|_{S}: S \rightarrow \partial M(G)$ is a conformal equivalence. Now let $\Gamma_{0}<\Gamma$ be a subgroup of index $d$. Then the inclusion $\Gamma_{0}$ in $\Gamma$ induces a branched covering map $p: M\left(\Gamma_{0}\right) \rightarrow M(\Gamma)$ of degree $d$, so that for any component $S \subset \partial M\left(\Gamma_{0}\right)$ the restriction $\left.p\right|_{S}$ is an orbifold covering map of degree $d$. Then $\pi \circ p$ is a non-simple holomorphic covering of degree $d$.

(2) Let $\Gamma$ be a Kleinian group such that $\partial M(\Gamma)$ is connected and the components of $\Omega(\Gamma)$ are simply connected with stabilizers of infinite index. Such groups are known as web-groups. Since geometrically finite Kleinian groups are also residually finite we can choose a subgroup $H<\Gamma$ of finite index 
such that $\partial M(H)$ is disconnected. The map $\pi: \partial M(H) \rightarrow \partial M(\Gamma)$ given by the canonical holomorphic orbifold covering induced by the inclusion $H \subset \Gamma$ is non-simple with at least two components $S_{1}$ and $S_{2} \subset \partial M(H)$ such that $\operatorname{deg}\left(\pi_{S_{1}}\right)>\operatorname{deg}\left(\left.\pi\right|_{S_{2}}\right)$.

We have not found in the literature whether for any connected hyperbolic Riemann surface $S$ there exists a web-group $G$ with $\partial M(G)$ conformally equivalent to $S$. However, M. Kapovich kindly pointed out that this construction can be done using the Brooks deformation theorem (see [5]).

Given a holomorphic covering $\left(R, S, S^{\prime}\right)$, if $S^{\prime}$ is connected, then we say that $R$ is primitive. We call a primitive holomorphic covering uniformizable if there exists a pair of web-groups $H<\Gamma$, with $H$ of finite index, so that the canonical holomorphic covering $\pi: M(H) \rightarrow M(\Gamma)$ belongs to $C H\left(R, S, S^{\prime}\right)$. So far we have no examples of non-simple non-uniformazible holomorphic coverings.

Connected sum of non-simple coverings. Given two primitive holomorphic coverings $\left(R_{1}, S_{1}, S_{1}^{\prime}\right)$ and $\left(R_{2}, S_{2}, S_{2}^{\prime}\right)$, we construct a connected sum $R_{1} \# R_{2}$, in a similar but slightly different way as in Subsection 3.1 as follows: for $i=1,2$ fix two open topological disks $t_{i} \subset S_{i}^{\prime}$, not containing branching points of $R_{i}$, respectively, together with a homeomorphism $\phi: \partial t_{1} \rightarrow \partial t_{2}$. Let $g_{1}$ be a single component of $R_{1}$; then the map $\phi \circ g_{1}$ defines a family of homeomorphisms from the components of $g_{1}^{-1}\left(\partial t_{1}\right)$ to $\partial t_{2}$. Let $S_{2}^{\prime \prime}=S_{2}^{\prime} \backslash t_{2}$ and we glue the copies of $S_{2}^{\prime \prime}$ to the surface $g_{1}^{-1}\left(S_{1}^{\prime} \backslash t_{1}\right)$ along the family of homeomorphisms $\phi \circ g_{1}$ on $g_{1}^{-1}\left(\partial t_{1}\right)$. Using induction with respect to all single components of $R_{1}$ we construct a non-simple holomorphic covering $\left(\hat{R}_{1}, T, T^{\prime}\right)$ with $T^{\prime}=S_{1}^{\prime} \# S_{2}^{\prime}$. Now repeat the process for a single component $g_{2}$ of $R_{2}$ to get a non-simple holomorphic covering $\left(\hat{R}_{2}, W, W^{\prime}\right)$ with $W^{\prime}=S_{1}^{\prime} \# S_{2}^{\prime}$ and finally we put $R_{1} \# R_{2}=\left(\hat{R}_{1}, T, T^{\prime}\right) \sqcup\left(\hat{R}_{2}, W, W^{\prime}\right)$ which is a primitive non-simple holomorphic covering.

Let us note that the case of holomorphic coverings over a surface of genus zero is special in the following sense: let $R_{1}$ and $R_{2}$ be non-simple holomorphic coverings onto the Riemann sphere with finitely many points removed. If $g$ is a single component of $R_{1}$, then the induced single component $f$ of $R_{1} \# R_{2}$ belongs to $H(g)$ up to forgetting additional perforations on the source and the target surfaces. We call such a primitive covering a primitive covering of genus zero. This observation leads to the following lemma.

Lemma 8. Let $R_{1}: S_{1} \rightarrow S_{1}^{\prime}$ and $R_{2}: S_{2} \rightarrow S_{2}^{\prime}$ be holomorphic coverings over $S_{1}^{\prime}$ and $S_{2}^{\prime}$ which are Riemann spheres with finitely many points removed. Then we can choose for $i=1,2$ disks $t_{i} \subset S_{i}^{\prime}$ and a gluing map $\phi: \partial t_{1} \rightarrow \partial t_{2}$ such that every single component of $R_{1} \# R_{2}$ belongs to either $C H\left(R_{1}\right)$ or $C H\left(R_{2}\right)$ up to forgetting additional perforations.

Proof. Let $t \subset \overline{\mathbb{C}}$ be a round open disk such that $t \subset S_{1}^{\prime}$ and not containing branching points of $R_{1}$. Let $X \subset \overline{\mathbb{C}}$ be the set $\overline{\mathbb{C}} \backslash\left(S_{2}^{\prime} \cup V\left(R_{2}\right)\right)$, where $V\left(R_{2}\right)$ is the set of branching points of $R_{2}$, and $\gamma \in P S L(2, \mathbb{C})$ is so that $\gamma(X) \subset t$. Then for the coverings $R_{1}$ and $\gamma \circ R_{2}$ we choose $t_{1}=t$ and $t_{2}=\overline{\mathbb{C}} \backslash t$. Taking $\phi=\operatorname{Id}$ on $\partial t$ finishes the proof of the lemma.

Another application of the Klein-Maskit combination theorem and the arguments of the proofs of Theorem 1 and Lemma 8 allow us to improve Theorem 1 as follows. 
Theorem 9. Let $R_{1}$ and $R_{2}$ be two primitive uniformizable genus zero holomorphic coverings. Then there exists a primitive uniformizible genus zero holomorphic covering $Q$ so that for every single component $q$ of $Q$ there exists $r$, which is either a single component of $R_{1}$ or a single component of $R_{2}$, so that $q \in C H(r)$ after, perhaps, forgetting additional perforations.

Proof. Let $\Gamma_{1}$ and $\Gamma_{2}$ be web-groups uniformizing $R_{1}$ and $R_{2}$. As in Theorem 1 we apply the Klein-Maskit combination theorem to $\Gamma_{1}$ and $\Gamma_{2}$ and construct a finite index subgroup of $\Gamma_{1} * \Gamma_{2}$ compatible to the connected sum $R_{1} \# R_{2}$.

Remark. The Klein-Maskit theorems are generalized to Kleinian groups in higher dimensions, so our Theorem 1 generalizes in that setting as well.

\section{Proof of Theorem 2}

Theorem 2 is a direct application of the Bers simultaneous uniformization theorem.

Proof of Theorem 2, Assume that $R_{1}$ and $R_{2}$ are two symmetric holomorphic coverings forming a simple hyperbolic cobordism. Then the respective geometrically finite Kleinian groups $\Gamma_{1}$ and $\Gamma_{2}$ are quasifuchsian. We can assume that $\Gamma_{1}<\Gamma_{2}$. Since every quasifuchsian group is quasiconformally equivalent to a Fuchsian group, $\Gamma_{2}$ admits an orientation reversing quasiconformal involution $\tau: \overline{\mathbb{C}} \rightarrow \overline{\mathbb{C}}$ commuting with $\Gamma_{2}$, interchanging components of $\Omega\left(\Gamma_{2}\right)$ and which is the identity on the limit set $\Lambda\left(\Gamma_{2}\right)$. Hence, $\tau$ commutes with $\Gamma_{1}$. Since $R_{1}$ and $R_{2}$ are symmetric, then $R_{1}$ and $R_{2}$ belong to the same Hurwitz class.

Assume that two symmetric holomorphic coverings $R_{1}$ and $R_{2}$ belong to the same Hurwitz class. Then we can construct cobordisms between $R_{1}$ and an anticonformal copy of $R_{1}$, together with a cobordism between $R_{2}$ and an anticonformal copy of $R_{2}$. The homeomorphisms $\phi$ and $\psi$ associated to $R_{1}$ and $R_{2}$ allow us to glue the given cobordisms along the anticonformal copies to get a cobordism between $R_{1}$ and $R_{2}$.

What follows is an example of a topological cobordism between the simplest rational maps.

Let us consider the convex combination between $z^{2}$ and $z^{3}$ :

$$
f_{t}(z)=(1-t) z^{2}+t z^{3}
$$

for $t \in I=[0,1]$. Then $f_{t}$ defines a rational endomorphism $F$ of 3-manifolds $X=\mathbb{C} \times I$ by the formula

Then

$$
F(z, t)=\left(f_{t}(z), t\right)
$$

i) The map $F$ is not a branched self-covering of $X$.

ii) Let $X_{t_{1}, t_{2}}=\mathbb{C} \times\left[t_{1}, t_{2}\right]$; then the restriction of $F$ to $X_{t_{1}, t_{2}}$ is a branched self-covering of $X_{t_{1}, t_{2}}$ for $0<t_{1}<t_{2}<1$.

iii) If $0<t_{1}<t_{2}<1$, then for $t_{1} \leq t \leq t_{2}$ the real polynomial $f_{t}$ is in general position and the sets of the critical values $\left\{v_{1}\right\},\left\{v_{2}\right\}$ of $F$ in $X_{t_{1}, t_{2}}$ forms two embedded arcs connecting the boundaries of $X_{t_{1}, t_{2}}$. Moreover, for each $i$ the set $F^{-1}\left(v_{i}\right)$ consists of two curves $\alpha_{i, j}$, with $j=1,2$, one is mapped homeomorphically onto the image by $F$, while the other consists of the critical points. Let $M=X_{t_{1}, t_{2}} \backslash\left(v_{1} \bigcup v_{2}\right)$ and $M=F^{-1}\left(M^{\prime}\right) \subset X_{t_{1}, t_{2}}$ be two 
3-manifolds; then $F: M \rightarrow M^{\prime}$ is a covering. The manifold $M$ is homeomorphic to the product of the five punctured sphere times a closed interval and the manifold $M^{\prime}$ is homeomorphic to the three punctured sphere times a closed interval. Since $f_{t_{i}}$ are symmetric maps and $f_{t_{2}} \in H\left(f_{t_{1}}\right)$, then by Theorem 2 the maps $f_{t_{1}}$ and $f_{t_{2}}$ are simply cobordant. Then on $M$ and $M^{\prime}$ there are hyperbolic structures depending on the extremes $t_{1}$ and $t_{2}$ such that $F$ is a Möbius morphism on $M$. This means that for $0<t_{1}<t_{2}<1$ we can define two hyperbolic 3-dimensional orbifold structures on which the map $F$ is a Möbius morphism.

iv) The endomorphism $F: X \rightarrow X$ is a Hausdorff limit (this is a particular case of Gromov-Hausdorff limit) of Möbius morphisms $F: X_{t_{1}, t_{2}} \rightarrow X_{t_{1}, t_{2}}$ for $t_{1} \rightarrow 0$ and $t_{2} \rightarrow 1$.

v) If $t \neq 0,1$, then we can obtain two functions $f_{0}$ and $f_{1}$ from the map $f_{t}$ using a pinching procedure with respect to peripheral curves. For the convenience of the reader we sketch this procedure when $f_{1}(z)=z^{3}$. Fix a $t \neq 0,1$ and a Jordan curve $\gamma \in \mathbb{C}$ so that $\alpha=f_{t}^{-1}(\gamma)$ is a connected Jordan curve. Then the finite critical values belong to the interior of $\gamma$. Let $A(\gamma)$ be an annular neighborhood of $\gamma$ so that $A(\alpha)=f_{t}^{-1}(A(\gamma))$ is an annular neighborhood of $\alpha$ and $f_{t}: A(\alpha) \rightarrow A(\gamma)$ is a covering of degree 3 . Let $\nu_{j}$ be a sequence of Beltrami differentials supported in $A(\gamma)$ as constructed in Section 2.1 and consider the extension of each $\nu_{j}$ on $\overline{\mathbb{C}}$ by zero outside $A(\gamma)$. Let $\mu_{j}(z)=v_{j}\left(f_{t}\right) \frac{\overline{f_{t}^{\prime}}}{f_{t}^{\prime}}$ be the pull-back of $\nu_{j}$ with respect to $f_{t}$. Let $\phi_{j}$ and $\psi_{j}$ be solutions of the Beltrami equation for $\mu_{j}$ and $\nu_{j}$, respectively, with $\phi_{j}(0)=\psi_{j}(0)=0, \phi_{j}(\infty)=\psi_{j}(\infty)=\infty$, and $\phi_{t}^{\prime}(\infty)=\psi_{j}^{\prime}(\infty)=1$. Then $p_{j}=\psi_{j} \circ f_{t} \circ \phi_{j}^{-1}$ are polynomials of degree 3 . Moreover, $\phi_{j}$ forms a family of univalent normalized holomorphic functions defined on a neighborhood of infinity $V$; then $\psi_{j}$ also forms a holomorphic family on $U=f_{t}^{-1}(V)$. After taking a suitable subsequence we can assume that $\phi_{j} \rightarrow \phi_{0}$ and $\psi_{j} \rightarrow \psi_{0}$ converge uniformly on compact subsets of $U$ and $V$, respectively. Moreover, $\phi_{0}$ and $\psi_{0}$ are non-constant functions. Hence $p_{j}$ converges to a degree 3 polynomial $p_{0}$ and, even more, $\left.p_{0}\right|_{\phi_{0}(U)}=\left.\psi_{0} \circ f_{t} \circ \phi_{0}^{-1}\right|_{\phi_{0}(U)}$. We claim that $p_{0}(z)=z^{3}$, otherwise, $p_{0}$ has a critical value $v_{0} \neq 0, \infty$. Thus $p_{j}$ also has a finite critical value $v_{j}$ converging to $v_{0}$. But $v_{j}=\psi_{j}\left(\frac{4}{27}(1-t)^{3} t^{2}\right)$ belongs to the bounded component of $\mathbb{C} \backslash\left\{\psi_{j}(A(\gamma))\right\}$. Since $\psi_{j}$ converges to $\psi_{0}$ and the moduli of the annuli $\psi_{j}(A(\gamma))$ converges to $\infty$, we have a contradiction to $v_{0} \neq 0$. Thus $p_{0}(z)=z^{3}$, as claimed.

In the case where $f_{0}(z)=z^{2}$, we consider a curve $\gamma$ closed to 0 so that $f_{t}^{-1}(\gamma)$ is the union of two curves $\alpha$ and $\beta$. Here $f_{t}$ is a degree 2 covering on $\alpha$, and $\beta$ belongs to the exterior of $\alpha$. Now we take the normalization of $\phi_{j}$ and $\psi_{j}$ given by $\phi_{j}(0)=\psi_{j}(0)=0, \phi_{j}^{\prime}(0)=\psi_{j}^{\prime}(0)=1$ and $\phi_{j}(\infty)=$ $\psi(\infty)=\infty$ and proceed as above.

vi) Now on $X_{t_{1}, t_{2}}$ with $0<t_{1}<t_{2}<1$ we put a quasiconformal deformation converging on the components of the boundary $\partial X_{t_{1}, t_{2}}$ to $z^{2}$ and $z^{3}$, respectively, to get a limit. This limit seems to be a sort of double limit converging in Hausdorff topology to a non-uniformizable object. This would give a non-geometric completion of the respective Teichmüller space. We suspect that any limit of this type belongs to $H(F, \mathbb{C} \times[0,1], \mathbb{C} \times[0,1])$. 
vii) Let $J\left(f_{t}\right)$ be the Julia set of $f_{t}$; then $J(F)=\bigcup_{t \in[0,1]}\left(J\left(f_{t}\right), t\right) \subset \mathbb{C} \times[0,1]$, is a closed set completely invariant under $F$. Computer experiments suggest that $\mathbb{C} \times I \backslash J(F)$ consists of two components. The one containing the set $\{0\} \times[0,1]$ is simply connected and the other one has infinitely generated fundamental group. The experiments also suggest that the set $J(F)$ is a non-locally connected embedding of $\mathbb{S}^{1} \times[0,1]$ with infinitely many cusps accumulating to a compact subset of the interior of $\mathbb{S}^{1} \times[0,1]$.

\section{Proof of Theorem 3}

As the previous discussion showed, the construction of cobordisms between rational maps often involves the introduction of single univalent components. In this chapter we look for a construction avoiding these components. We call this type of construction a pure cobordism.

Let $\mathbb{D}_{\infty}$ be the completion of $\mathbb{C}$ by adding the circle at infinity $\infty \cdot \mathbb{S}^{1}$. Then any monic holomorphic polynomial $P$ of degree $d$ can be extended to the circle at infinity by the formula $\infty \cdot e^{i \theta} \mapsto \infty \cdot e^{d i \theta}$ so that $P$ defines a branched self-covering $\hat{P}$ of $\mathbb{D}_{\infty}$ and $\left.\hat{P}\right|_{\partial \mathbb{D}_{\infty}}(z)=z^{d}$. Now we identify $\mathbb{D}_{\infty}$ with the unit disk $\mathbb{D} \subset \mathbb{C}$. Let us consider two monic polynomials $P_{1}$ and $P_{2}$, acting on $\mathbb{C}$, then define a finite degree branched covering $F: \overline{\mathbb{C}} \rightarrow \overline{\mathbb{C}}$ as follows:

$$
F(z)= \begin{cases}\hat{P}_{1}(z) & \text { for } z \in \mathbb{D}, \\ \gamma \circ \hat{P}_{2} \circ \gamma(z) & \text { for } z \in \overline{\mathbb{C}} \backslash \mathbb{D}, \gamma(z)=\frac{1}{\bar{z}} .\end{cases}
$$

In holomorphic dynamics the map $F$ is known as the formal mating of the monic polynomials $P_{1}$ and $P_{2}$ (see for example [9] or [11]).

Now we need the following theorem.

Theorem 10. We have two polynomials $P_{1}$ and $P_{2}$ of the same degree $d>2$ with canonical holomorphic representatives. Assume one of them, say $P_{2}$, is symmetric; then there exists a rational map $R$ such that the following holomorphic coverings form a hyperbolic cobordism:

$$
\begin{aligned}
& \text { - } P_{1}: \mathbb{C} \backslash\left(P_{1}^{-1}\left(V\left(P_{1}\right)\right)\right) \rightarrow \mathbb{C} \backslash V\left(P_{1}\right), \\
& \text { - } P_{2}: \mathbb{C} \backslash\left(P_{2}^{-1}\left(V\left(P_{2}\right)\right)\right) \rightarrow \mathbb{C} \backslash V\left(P_{2}\right), \\
& \text { - } R: \mathbb{C} \backslash\left(R^{-1}(V(R))\right) \rightarrow \mathbb{C} \backslash V(R) .
\end{aligned}
$$

Proof. Given two polynomials $P_{1}$ and $P_{2}$ as in the statement of the theorem, we can assume that $P_{1}$ and $P_{2}$ are monic. Let us consider their formal mating $F$. Let $Q \in H(F, \overline{\mathbb{C}}, \overline{\mathbb{C}}) \cap$ Rat be a rational map. First note that since $F^{-1}(\partial \mathbb{D})=\partial \mathbb{D}$, then there is a Jordan curve $\delta \subset \overline{\mathbb{C}}$ such that $Q^{-1}(\delta)$ is a connected Jordan curve. If $V(Q)$ is the set of critical values of $Q$, then $Q$ is a holomorphic covering of finite degree from $S_{1}=\overline{\mathbb{C}} \backslash Q^{-1}(V(Q))$ to $S_{2}=\overline{\mathbb{C}} \backslash V(Q)$.

Let $\Gamma_{1}<\Gamma_{2}$ be two finitely generated Fuchsian groups uniformizing $Q: S_{1} \rightarrow$ $S_{2}$ in $\mathbb{D}$. Then we can assume that the map $R: \mathbb{D}^{*} / \Gamma_{1} \rightarrow \mathbb{D}^{*} / \Gamma_{2}$ induced by the inclusion is a rational map satisfying $R(z)=\overline{Q(\bar{z})}$. We claim that the triple $\left(R, P_{1}, P_{2}\right)$ forms a cobordant family.

Indeed, let us apply a pinching procedure to the covering $Q$, with respect to the curve $\delta$. Then by Theorem 4 we obtain a Kleinian group $\Gamma_{2, \infty}$ with an isomorphism $\rho: \Gamma_{2} \rightarrow \Gamma_{2, \infty}$ so that $\Gamma_{2, \infty}$ is a geometrically finite function group with $S\left(\Gamma_{2, \infty}\right)=$ $S_{2}^{\prime} \sqcup T_{2}^{\prime} \sqcup T_{2}^{\prime \prime}$ where $S_{2}^{\prime}$ is anticonformally equivalent to $S_{2}$ and $T_{2}^{\prime}$ and $T_{2}^{\prime \prime}$ are finitely 
punctured Riemannian spheres. Each of them contains only one accidental cusp, that is, determined by $\delta$.

On the other hand, we have a geometrically finite function group $\Gamma_{1, \infty}$ satisfying $\Gamma_{1, \infty}<\Gamma_{2, \infty}$. In particular, since $R^{-1}(\delta)$ is connected, then $\Gamma_{1, \infty}$ also is presented by pinching the group $\Gamma_{1}$ with respect to the curve $R^{-1}(\delta)$ and contains only one conjugacy class of an accidental parabolic element. Hence $S\left(\Gamma_{1, \infty}\right)=S_{1}^{\prime} \sqcup T_{1}^{\prime} \sqcup T_{1}^{\prime \prime}$, where $S_{1}^{\prime}$ is an anticonformal copy of $S_{1}$ and the surfaces $T_{1}^{\prime}$ and $T_{1}^{\prime \prime}$ are finitely punctured spheres containing only one accidental cusp determined by $R^{-1}(\delta)$. This implies that the map $\alpha$ induced by inclusion is a holomorphic simple covering from $S\left(\Gamma_{1, \infty}\right)$ to $S\left(\Gamma_{2, \infty}\right)$ so that $\left.\alpha\right|_{S_{1}^{\prime}}: S_{1}^{\prime} \rightarrow S_{2}^{\prime}$ belongs to $H(R)$. As the preimage of an accidental cusp is an accidental cusp, then the maps $\left.\alpha\right|_{T_{1}^{\prime}}$ and $\left.\alpha\right|_{T_{1}^{\prime \prime}}$ are in the Hurwitz conformal classes of some polynomials, say $Q_{1}$ and $Q_{2}$, respectively. By construction $Q_{1} \in H\left(P_{1}\right)$ and $Q_{2} \in H\left(P_{2}\right)$ with orientation reversing homeomorphisms $\phi_{2}$ and $\psi_{2}$. Since $P_{2}$ is symmetric, then the homeomorphisms $\phi_{2}$ and $\psi_{2}$ also can be chosen to be quasiconformal orientation preserving homeomorphisms. If $\phi_{i} \circ Q_{i}=P_{i} \circ \psi_{i}$, then let $\mu$ be the Beltrami differential on $S_{2}^{\prime} \sqcup T_{2}^{\prime} \sqcup T_{2}^{\prime \prime}$ given in local coordinates by

$$
\mu(z)= \begin{cases}\frac{\bar{\partial} \phi_{1}}{\partial \phi_{1}}(z) & \text { on } T_{2}^{\prime}, \\ \frac{\partial \phi_{2}}{\partial \phi_{2}}(z) & \text { on } T_{2}^{\prime \prime}, \\ 0 & \text { on } S_{2}^{\prime},\end{cases}
$$

and let $\nu$ be the pull-back of $\mu$ by the orbit projection

$$
\pi_{2}: \Omega\left(\Gamma_{2, \infty}\right) \rightarrow \Omega\left(\Gamma_{2, \infty}\right) / \Gamma_{2, \infty}
$$

If $f_{\nu}$ is a solution of the Beltrami equation with respect to $\nu$, then the groups $G_{1}=f_{\nu} \circ \Gamma_{1, \infty} \circ f_{\nu}^{-1}$ and $G_{2}=f_{\nu} \circ \Gamma_{2, \infty} \circ f_{\nu}^{-1}$ satisfy our claim and finish the proof of the theorem.

Let us note that, by Theorem 4, each of the manifolds $M\left(G_{1}\right)$ and $M\left(G_{2}\right)$, constructed in the proof of Theorem 10, is homeomorphic to a set $U$ which is the complement of two open round 3-dimensional balls $B_{1}$ and $B_{2}$ in the unit ball $B$ with finitely many embedded arcs, connecting the boundary components of $\partial U$, removed. See Figure 3 ,

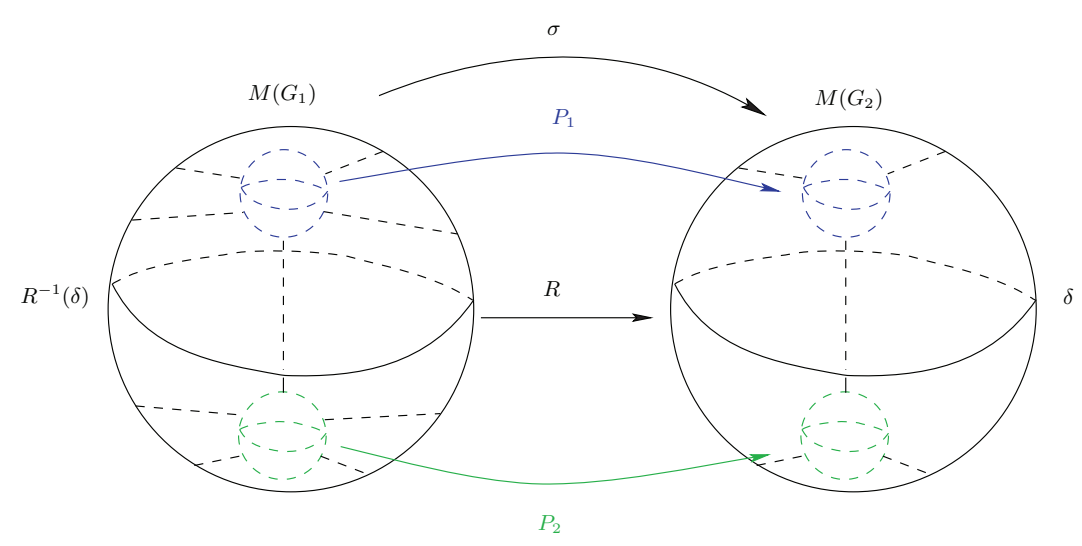

Figure 3. A sketch of the manifolds $M\left(G_{1}\right)$ and $M\left(G_{2}\right)$. 
The map $\sigma: M\left(G_{1}\right) \rightarrow M\left(G_{2}\right)$ induced by the inclusion $G_{1}<G_{2}$ can be extended on $U$ as a finite degree branched covering $\sigma^{*}: U \rightarrow U$ so that in suitable coordinates $\left.\sigma^{*}\right|_{\partial U} \in C H(R, \overline{\mathbb{C}}, \overline{\mathbb{C}}) \cup C H\left(P_{1}, \overline{\mathbb{C}}, \overline{\mathbb{C}}\right) \cup C H\left(P_{2}, \overline{\mathbb{C}}, \overline{\mathbb{C}}\right)$. In other words, in $U$ there are two Möbius orbifold structures which makes $\sigma^{*}$ a Möbius morphism.

Now if $\tau$ is the reflection of $S^{3}$ with respect to the unit sphere, then on $W=$ $U \cup \tau(U)$ we can extend $\sigma^{*}$ to a finite degree self-covering of $W$ by putting

$$
\Sigma(z)= \begin{cases}\sigma^{*}(z) & \text { on } U, \\ \tau \circ \sigma^{*} \circ \tau(z), & \text { on } \tau(U) .\end{cases}
$$

Then $\Sigma$ serves as a topological cobordism between $P_{1} \sqcup P_{2}$ and its anticonformal copies. In what follows, we will show that on $W$ there are two Möbius structures under which $\Sigma$ is a Möbius morphism. The idea is to apply the arguments of the proof of the Brooks orbifold deformation theorem to put on $M\left(G_{2}\right)$ a non-orientable uniformazible orbifold structure $\omega$ so that the component $S_{0} \subset \partial M\left(G_{2}\right)$ corresponding to the unit sphere consists of the "interior points" in the orbifold structure $\omega$ and other components of $\partial M\left(G_{2}\right)$ equipped with $\omega$ are Möbius equivalent to the previous structure.

Then there exists a degree 2 covering $\beta: X \rightarrow\left(M\left(G_{2}\right), \omega\right)$ such that $X$ is an orientable hyperbolic 3-orbifold with four boundary components which are mapped univalently by $\beta$ onto $\partial M\left(G_{2}\right) \backslash S_{0}$.

The following statement is the main lemma of this section, which is an application of arguments of the proof of the Brooks orbifold deformation theorem (see [5]).

Lemma 11. Let $\Gamma$ be a geometrically finite Kleinian group without torsion. Let $T \subset S(\Gamma)$ be a proper subcollection of surfaces. Then there exists a geometrically finite Kleinian group $G$ such that $S(G)$ is conformally equivalent to $T \sqcup T^{*}$ where $T^{*}$ is an anticonformal copy of $T$.

Proof. Let $T^{\prime}$ be the complement of the collection $T$ in $S(\Gamma)$. Our goal is to destroy the non-empty collection $T^{\prime}$. First, assume that there exists a round disk pattern $K$ covering just $T^{\prime}$. Let $\Gamma_{K}$ be the group generated by $\Gamma$ and the reflections with respect to all the disks projecting onto the elements of $K$ as in the discussion before Theorem 6. Then by Theorem 6 the hyperbolic orbifold $M\left(\Gamma_{K}\right)$ has as underlying space, the manifold $M_{K}$, where $M_{K}$ is homeomorphic to $M(\Gamma)$. Let $G_{K}<\Gamma_{K}$ be the subgroup of orientation preserving elements of $\Gamma_{K}$; then $G_{K}=\Gamma_{K} \cap P S L(2, \mathbb{C})$ is a normal order two subgroup of $\Gamma_{K}$ containing $\Gamma$. By the remark after Theorem 6. if a component $\Omega_{0} \in \Omega(\Gamma)$ covers an element of $T$, then the stabilizer of $\Omega_{0}$ in $G_{K}$ coincides with the stabilizer of $\Omega_{0}$ in $\Gamma$. Then $M\left(G_{K}\right)$ admits an anticonformal involution $\tau$ and $M\left(\Gamma_{K}\right)=M\left(G_{K}\right) / \tau$. Hence $\partial M\left(G_{K}\right)=T \sqcup T^{*}$, where $T^{*}$ is an anticonformal copy of $T$.

To finish the proof we have to justify the existence of the pattern $K$. By Theorem 7 there exists a quasiconformal homeomorphism $h$ and a group $\Gamma_{h}=h \circ \Gamma \circ h^{-1}$ admitting a disk pattern covering the whole surface $S\left(\Gamma_{h}\right)$.

Let us consider a subpattern $K^{\prime} \subset K$ precisely covering the collection $h\left(T^{\prime}\right) \subset$ $S\left(\Gamma_{h}\right)$ and construct a group $G_{h}$ which uniformizes the surfaces $S\left(G_{h}\right)=h(T) \sqcup$ $(h(T))^{*}$. Taking a suitable quasiconformal deformation for $G_{h}$ we obtain a group $G$, as claimed.

The lemma above allows to produce a hyperbolic orbifold structure on a double of the manifold $M(\Gamma)$ with respect to a complementary collection $T^{\prime}$. In other 
words, with this lemma one can endow an orbifold structure on the manifold $W$. The following theorem shows that we can put other orbifold hyperbolic structures on $W$ in such a way that the map $\Sigma$ becomes a Möbius morphism between these structures.

Theorem 12. The family of canonical holomorphic representatives of any collection of four polynomials in general position of the same degree $d>2$ forms a hyperbolic cobordism.

Proof. Take any pair of polynomials $P_{1}$ and $P_{2}$ from the given four. By Theorem 10. there exists a rational map $R$ such that $R \sqcup P_{1} \sqcup P_{2}$ forms a cobordant family of coverings. Let $\Gamma_{1}<\Gamma_{2}$ be the Kleinian groups realizing this cobordism, that is, the $\alpha: M_{\Gamma_{1}} \rightarrow M_{\Gamma_{2}}$ so that $\alpha$ maps $S\left(\Gamma_{1}\right)=V_{0} \sqcup V_{1} \sqcup V_{2}$ onto $S\left(\Gamma_{2}\right)=U_{0} \sqcup U_{1} \sqcup U_{2}$ and $\left(\alpha, \partial M\left(\Gamma_{1}\right), \partial M\left(\Gamma_{2}\right)\right)$ belongs to the Hurwitz class $C H\left(R, P_{1}, P_{2}\right)$. Let $H_{2}$ be the geometrically finite group with $S\left(H_{2}\right)=\left(U_{1} \sqcup U_{2}\right) \sqcup\left(\left(U_{1}\right)^{*} \sqcup\left(U_{2}\right)^{*}\right)$ given by Lemma 11] here again $\left(U_{1}\right)^{*}$ and $\left(U_{2}\right)^{*}$ are anticonformal copies of $U_{1}$ and $U_{2}$, respectively.

We claim that there exists a finite index subgroup $H_{1}<H_{2}$ with $S\left(H_{1}\right)=$ $\left(V_{1} \sqcup V_{2}\right) \sqcup\left(\left(V_{1}\right)^{*} \sqcup\left(V_{2}\right)^{*}\right)$ and a projection induced by inclusion of groups

$$
\beta: M\left(H_{1}\right) \rightarrow M\left(H_{2}\right)
$$

is so that $\left.\beta\right|_{V_{i}}$ is conformally equivalent to $P_{i}$, for $i=1,2$, and $\left.\beta\right|_{\left(V_{i}\right)^{*}}$ are anticonformal copies of $P_{i}$, respectively. Indeed, by the Brooks orbifold deformation theorem as used in Lemma 11 we can assume that the group $\Gamma_{2}$ admits a pattern $K$ which covers only the surface $U_{0}$.

Since $\alpha$ is a Möbius morphism, then $K^{\prime}=\alpha^{-1}(K) \subset V_{0}$ is also a pattern on $S\left(\Gamma_{1}\right)$ completely covering just the surface $V_{0}$. Hence the group $G_{1}$, generated by $\Gamma_{1}$ and the reflections with respect to the boundaries of all round disks which project on all elements of $K^{\prime}$, is a finite index subgroup of the group $G_{2}$, where $G_{2}$ is generated by $\Gamma_{2}$ and the reflections with respect to the boundaries of all disks projecting on all elements of $K$. In fact, these families of disks for $\Gamma_{1}$ and $\Gamma_{2}$ coincide.

Therefore the orientation preserving subgroups $H_{1}=G_{1} \cap P S L(2, \mathbb{C})$ and $H_{2}=$ $G_{2} \cap P S L(2, \mathbb{C})$ are geometrically finite Kleinian groups such that $H_{1}$ has finite index in $H_{2}$. Then the groups $H_{1}<H_{2}$ are the desired groups, as claimed.

Let us note that the anticonformal copies of $P_{1}$ and $P_{2}$ belong to the Hurwitz classes of the polynomials $P_{3}$ and $P_{4}$. Indeed all polynomials in general position, of the same degree, belong to the same Hurwitz class. Hence, after a suitable quasiconformal deformation of the pair $H_{1}$ and $H_{2}$ we complete the proof of the theorem.

Choose a surface $S \subset S\left(G_{2}\right)$ together with a pattern precisely covering $S$. Repeating the construction above, we construct a cobordism between the canonical representatives of six polynomials of the same degree in general position. The iteration of this procedure shows the following statement.

If the canonical holomorphic representatives of a collection of polynomials $P_{1}, \ldots, P_{n}$ in general position of the same degree $d>2$ forms a hyperbolic cobordism, then for every $k<n$, the canonical holomorphic representatives of every collection of $2(n-k)$ polynomials in general position and of degree $d$ forms a hyperbolically cobordant family. An induction argument over Theorems 12 and 2 completes the proof of Theorem 3 . 
Remark. Unfortunately, we were not able to prove the following desirable statement:

Every finite collection of non-univalent rational (polynomial) maps forms a cobordant family.

This is not clear even in the case of a single rational map $R$ (for more details on this problem see 3 ).

But if we drop the geometrically finiteness condition in the definition of cobordism, then, as it was shown in [3], in the case of a single rational map $R$ the statement above is always true, the corresponding uniformizing group is totally degenerated, and the respective Möbius morphism is Hurwitz equivalent to the radial extension of $R$ in the unit 3-dimensional ball.

\section{On CONFOrmal HuRwitz Classes AND SANDWich SEMigroups}

According to the discussion above it is interesting to know when two given rational maps belong to the same conformal or anticonformal Hurwitz class or, better, when these maps are conformally or anticonformally conjugated. It turns out that the answer is purely algebraic and does not require any dynamical information. We give a precise answer using sandwich products induced by the given rational maps. Also we suggest another point of view on Hurwitz classes of rational maps as minimal representation spaces of semigroups of holomorphic correspondences associated to the given holomorphic coverings.

The results in this section develop ideas in [3], and the Schreier representation of semigroups as treated in [2. We start with a brief introduction to Schreier representations.

Definition. Let $X$ be a topological space and let $\operatorname{End}(X)$ be the semigroup of continuous endomorphisms of the space $X$. Then

(1) $\operatorname{End}(X)$ is a topological semigroup.

(2) $X$ canonically embeds in $\operatorname{End}(X)$ as the ideal $I$ of constant endomorphisms.

(3) $I$ is the unique minimal bi-ideal (left and right) consisting of idempotents.

(4) (Schreier lemma) Let $G<\operatorname{End}(X)$ be a subsemigroup with $G \cap I=A \neq \emptyset$ and let $\rho: G \rightarrow \operatorname{End}(X)$ be a homomorphism. Then there exists a map $f: A \rightarrow X$ such that $f(g(x))=\rho(g)(f(x))$ for all $x \in A$ and $g \in G$, here $f(x):=\rho(x)$. In other words, every homomorphism is generated by a map. Even more, the map $f$ is continuous if and only if $\rho$ is continuous, and $f$ is a homeomorphism if and only if $\rho$ is a continuous isomorphism onto its image. We say that $\rho$ is orientation preserving or non-orientable, depending on whether $f$ has the corresponding property.

(5) Let $f: Y \rightarrow X$ be a continuous map between topological spaces. Then the set $G$ of all continuous maps $g: X \rightarrow Y$ can be transformed into a semigroup with the product:

$$
g_{1} *_{f} g_{2}=g_{1} \circ f \circ g_{2} .
$$

This product is called the sandwich product with respect to $f$ and $G_{f}=$ $\left\langle G, *_{f}\right\rangle$ is called the sandwich semigroup. If $f$ is not invertible, then $G_{f}$ does not contain a unit.

The following theorem appears in [2]. For convenience we include the proof.

Theorem 13. Let $R_{1}: \overline{\mathbb{C}} \rightarrow \overline{\mathbb{C}}$ and $R_{2}: \overline{\mathbb{C}} \rightarrow \overline{\mathbb{C}}$ be two rational maps, and let $G_{1}$ and $G_{2}$ be sandwich semigroups of rational maps with respect to $R_{1}$ and 
$R_{2}$, respectively. If $\rho: G_{1} \rightarrow G_{2}$ is an isomorphism, then there exist an element $\gamma \in P S L(2, \mathbb{C})$, and a bijection $\phi: \overline{\mathbb{C}} \rightarrow \overline{\mathbb{C}}$ so that $\rho(R)=\phi \circ R \circ \phi^{-1} \circ \gamma$ for every rational map $R$.

We say that the homomorphism $\rho$ is orientation preserving or non-orientable depending on whether $\phi$ has the same property.

Proof. Let $f=\left.\rho\right|_{\mathbb{C}}$ be the restriction of $\rho$ to the constants; then $f(\overline{\mathbb{C}}) \subset \overline{\mathbb{C}}$. Indeed for a suitable constant $c \in \overline{\mathbb{C}}$ then $c * R_{1} Q=c$ and hence $\rho(c) * R_{2} \rho(Q)=\rho(c)$ for every rational map $Q$. Since $\rho$ is an isomorphism $\rho(Q)$, then $\rho(c)$ is a constant. Also $f$ is a bijection.

Now we show that $\rho(P S L(2, \mathbb{C}))=P S L(2, \mathbb{C})$. Indeed, since for every rational maps $R$ and $Q$ we have $\rho\left(R *_{R_{1}} Q\right)=\rho(R) *_{R_{2}} \rho(Q)$, taking $Q=c$ a constant then $f\left(R \circ R_{1}(c)\right)=\rho(R) \circ R_{2}(f(c))$. As $f$ is invertible we have $\operatorname{deg}\left(R \circ R_{1}\right)=$ $\operatorname{deg}\left(\rho(R) \circ R_{2}\right)$. Similarly for $\rho^{-1}$ we have

$$
f^{-1}\left(R \circ R_{2}(c)\right)=\rho^{-1}(R) \circ R_{1}\left(f^{-1}(c)\right)
$$

and

$$
\operatorname{deg}\left(R \circ R_{2}\right)=\operatorname{deg}\left(\rho^{-1}(R) \circ R_{1}\right) .
$$

If $R \in P S L(2, \mathbb{C})$, then $\operatorname{deg}\left(R_{1}\right)=\operatorname{deg}\left(R \circ R_{1}\right)=\operatorname{deg}(\rho(R)) \cdot \operatorname{deg}\left(R_{2}\right)=\operatorname{deg}(\rho(R))$. $\operatorname{deg}\left(\rho^{-1}(R)\right) \cdot \operatorname{deg}\left(R_{1}\right)$ which implies $\operatorname{deg}(\rho(R))=1$.

Let $\gamma=\rho(\mathrm{Id})$ so $\gamma \in P S L(2, \mathbb{C})$. Consider the map $\tau_{\gamma}: G_{2} \rightarrow\left\langle\right.$ Rat, $\left.*_{\gamma \circ R_{2}}\right\rangle$ given by $\tau_{\gamma}(R)=R \circ \gamma^{-1}$. Then $\tau_{\gamma}$ is an isomorphism of the sandwich semigroup, which follows from direct computation:

$$
\tau_{\gamma}\left(R *_{R_{2}} Q\right)=\tau_{\gamma}\left(R \circ R_{2} \circ Q\right)=R \circ R_{2} \circ Q \circ \gamma^{-1}=\tau_{\gamma}(R) *_{\gamma \circ R_{2}} \tau_{\gamma}(Q) .
$$

Then $\Phi: \tau_{\gamma} \circ \rho: G_{1} \rightarrow\left\langle\right.$ Rat, $\left.*_{\gamma \circ R_{2}}\right\rangle$ is an isomorphism satisfying $\Phi(\mathrm{Id})=$ Id . If $R=c$ is a constant, then $\Phi(c)=\rho(c) \circ \gamma=\rho(c)=f(c)$. Also $\Phi\left(R_{1}\right)=\Phi\left(\operatorname{Id} *_{R_{1}} \operatorname{Id}\right)=$ $\gamma \circ R_{2}$, and hence for every $c \in \mathbb{C}$

$$
f\left(R_{1}(c)\right)=\Phi\left(\operatorname{Id} *_{R_{1}} c\right)=\gamma \circ R_{2}(f(c))
$$

which implies $R_{2}=\gamma^{-1} \circ f \circ R_{1} \circ f^{-1}$. We have for every $Q \in \operatorname{Rat}(\mathbb{C})$ and $c \in \mathbb{C}$,

$$
f\left(Q \circ R_{1}(c)\right)=\Phi\left(Q *_{R_{1}} c\right)=\Phi(Q) \circ \gamma \circ R_{2}(f(c))=\Phi(Q) \circ f \circ R_{1}(c) .
$$

Then

$$
Q \circ R_{1}(c)=f^{-1} \circ \Phi(Q) \circ f \circ R_{1}(c)
$$

and so

$$
\Phi(Q)=f \circ Q \circ f^{-1}
$$

but $\Phi(Q)=\tau_{\gamma} \circ \rho(Q)$ or $\rho(Q)=\Phi(Q) \circ \gamma$, as we wanted to prove.

As an immediate corollary we have the following.

Corollary 14. If $\rho: G_{1} \rightarrow G_{2}$ is an isomorphism as in Theorem 12 and $\rho(\mathrm{Id})=\mathrm{Id}$, then there exists a bijection $f: \overline{\mathbb{C}} \rightarrow \overline{\mathbb{C}}$ such that

$$
\rho(R)=f \circ R \circ f^{-1} .
$$

The next theorem is the main result of this section. 
Theorem 15. Let $R_{1}$ and $R_{2}$ be non-constant rational maps and let $G_{1}$ and $G_{2}$ be the respective sandwich semigroups on $\operatorname{Rat}(\mathbb{C})$. Then

(1) The pair $R_{1}, R_{2}$ belongs to the same conformal Hurwitz class if and only if there exists a continuous orientation preserving isomorphism $\rho: G_{1} \rightarrow G_{2}$. Moreover, $\rho(\mathrm{Id})=\mathrm{Id}$ if and only if $R_{1}$ is $P S L(2, \mathbb{C})$ conjugated to $R_{2}$.

(2) A continuous isomorphism $\rho$ reverses orientation if and only if $R_{1}$ is an anticonformal copy of $R_{2}$. Moreover $\rho(\mathrm{Id})=\mathrm{Id}$ if and only if $R_{1}$ is anticonformally conjugated to $R_{2}$.

Proof. Part 1. Assume that $R_{1}$ and $R_{2}$ belong to the same conformal Hurwitz class and let $h, g \in P S L(2, \mathbb{C})$ be so that $R_{2}=g^{-1} \circ R_{1} \circ h$. Then the map $\rho(R)=h^{-1} \circ R \circ g$ defines a continuous orientation preserving isomorphism from $G_{1}$ to $G_{2}$. Indeed, from direct calculation:

$$
\begin{gathered}
\rho\left(R *_{R_{1}} Q\right)=h^{-1} \circ R \circ R_{1} \circ Q \circ g \\
=\rho(R) *_{R_{2}} \rho(Q) .
\end{gathered}
$$

Now if $\rho: G_{1} \rightarrow G_{2}$ is an orientation preserving isomorphism, then by Theorem 12 there exists a $\gamma \in P S L(2, \mathbb{C})$ and a bijection $f: \overline{\mathbb{C}} \rightarrow \overline{\mathbb{C}}$ so that $\rho(Q)=f \circ Q \circ f^{-1} \circ \gamma$ for every rational map $Q$. By conjugation, $f$ defines an automorphism of $\operatorname{Rat}(\mathbb{C})$ with composition as a product. By Proposition 8 in [2], the map $f$ belongs to the group generated by $P S L(2, \mathbb{C})$ and the absolute Galois group. Since $\rho$ is continuous and orientation preserving we have $f \in P S L(2, \mathbb{C})$. Now assume $\rho(\mathrm{Id})=\mathrm{Id}$; then by Corollary [14, $\rho(R)=f \circ R \circ f^{-1}$ and $f \in P S L(2, \mathbb{C})$ and $\rho\left(R_{1}\right)=f \circ R_{1} \circ f^{-1}=$ $\rho\left(\mathrm{Id} *_{R_{1}} \mathrm{Id}\right)=R_{2}$.

Part 2. If $\rho$ is continuous and orientation reversing, then $\bar{f} \in P S L(2, \mathbb{C})$ and the proof goes as Part 1.

In conclusion we note the following.

First, it is possible to show that every continuous semigroup product on $\operatorname{Rat}(\mathbb{C})$ which is continuously isomorphic to a sandwich product is a sandwich product itself. So the classes of continuous isomorphisms of sandwich semigroups on $\operatorname{Rat}(C)$ correspond to the conformal Hurwitz classes of rational maps.

Second, that it is not clear at all how to associate the algebraic characterization of the conformal Hurwitz class of symmetric rational maps with the geometric cobordisms point of view.

Finally, similar ideas allow us to consider the Hurwitz space as a representation space of a special class of holomorphic correspondences. This follows using results from [2] with [3]. So we can construct a Teichmüller space of correspondences of the form $R^{-1} \circ R$, called the deck correspondence associated to $R$. From [3] it follows that the Speisser class of $R$ fibers over the moduli space of the deck with fiber equivalent to the conformal Hurwitz class of $R$. Let $G_{R}=\left\langle R^{-1} \circ R, \overline{\mathbb{C}}\right\rangle$ be the semigroup of holomorphic correspondences generated by the deck correspondence associated to $R$ and constant maps. Consider the space $\mathcal{X}$ of all representations of $G_{R}$ into the semigroup of holomorphic correspondences on $\overline{\mathbb{C}}$. Then using results from [2], one can consider the Speisser class of a rational map $R$ as a subspace of the connected component of $\mathcal{X}$ containing the identity representation. 


\section{ACKNOWLEDGMENTS}

The authors would like to thank M. Kapovich for pointing out the ideas in the Brooks deformation theorem which simplified a previous version of the proof of Theorem 3. Also we would like to thank the referee for useful comments.

\section{REFERENCES}

[1] Israel Berstein and Allan L. Edmonds, On the construction of branched coverings of lowdimensional manifolds, Trans. Amer. Math. Soc. 247 (1979), 87-124, DOI 10.2307/1998776. MR 517687

[2] Carlos Cabrera, Peter Makienko, and Peter Plaumann, Semigroup representations in holomorphic dynamics, Discrete Contin. Dyn. Syst. 33 (2013), no. 4, 1333-1349, DOI 10.3934/dcds.2013.33.1333. MR2995848

[3] Carlos Cabrera, Peter Makienko, and Guillermo Sienra, On Poincaré extensions of rational maps, Conform. Geom. Dyn. 19 (2015), 197-220, DOI 10.1090/ecgd/281. MR.3373954

[4] A. È. Erëmenko and M. Yu. Lyubich, Dynamical properties of some classes of entire functions (English, with English and French summaries), Ann. Inst. Fourier (Grenoble) 42 (1992), no. 4, 989-1020. MR.1196102

[5] Michael Kapovich, Hyperbolic manifolds and discrete groups, Modern Birkhäuser Classics, Birkhäuser Boston, Inc., Boston, MA, 2009. Reprint of the 2001 edition. MR2553578

[6] Albert Marden, Hyperbolic manifolds, Cambridge University Press, Cambridge, 2016. An introduction in 2 and 3 dimensions. MR 3586015

[7] Bernard Maskit, On Klein's combination theorem. II, Trans. Amer. Math. Soc. 131 (1968), 32-39, DOI 10.2307/1994678. MR223570

[8] Bernard Maskit, Kleinian groups, Grundlehren der Mathematischen Wissenschaften [Fundamental Principles of Mathematical Sciences], vol. 287, Springer-Verlag, Berlin, 1988. MR 959135

[9] John Milnor, Pasting together Julia sets: a worked out example of mating, Experiment. Math. 13 (2004), no. 1, 55-92. MR2065568

[10] Ken'ichi Ohshika, Geometrically finite Kleinian groups and parabolic elements, Proc. Edinburgh Math. Soc. (2) 41 (1998), no. 1, 141-159, DOI 10.1017/S0013091500019477. MR.1604337

[11] Lei Tan, Matings of quadratic polynomials, Ergodic Theory Dynam. Systems 12 (1992), no. 3, 589-620, DOI 10.1017/S0143385700006957. MR.1182664

Instituto de Matematicas, Unidad Cuernavaca, University Nacional Autonoma de Mexico, Av Universidad s/n, Col Lomas de Chamilpa, 62210 Cuernavaca, MOR, Mexico

Email address: carloscabrerao@im.unam.mx

Instituto de Matematicas, Unidad Cuernavaca, University NaCional Autonoma de Mexico, Av Universidad s/n, Col Lomas de Chamilpa, 62210 Cuernavaca, MOR, Mexico

Email address: makienko@im.unam.mx

Facultad de Ciencias, Universidad Nacional Autonoma De Mexico, Av. Universidad 3000, 04510 MEXICO

Email address: gsl@dinamica1.fciencias.unam.mx 